\title{
Wideband One Way Antenna without Grounding
}

\author{
Michael Bank \\ Telecommunication System Engineering, Jerusalem College of Technology, Jerusalem 91160, Israel
}

\begin{abstract}
This article proposes a new type of antenna which allows getting rid of certain limitations of classic monopole antenna. Like a normal monopole, this antenna belongs to a class of radio antenna consisting of a straight rod-shaped conductor. But unlike a classic monopole, this antenna can operate at all frequencies of a very wide range. In addition, it does not require grounding. The article considers the wide possibilities of antenna applications.
\end{abstract}

Key words: Dipole, monopole, MB antenna, inverter, WBMB antenna, S11, gain, folded dipole, magnetic antenna, antenna reactive resistance, transparent antenna, nullifier, circular polarization, single line.

\section{Introduction}

Radio engineering developed parallel to the research of the theory of electromagnetic oscillations and inventions of new principles of the equipment. The most important inventions are: the coherent detector of Brandly (1892); radio receiver of Popov, Marconi et al. (1895); biconical antenna of Lodge (1898); sine-wave transmitter of Poulsen (1903); superheterodyne receiver of Armstrong (1918); homodyne receiver of Colebrook (1924); and frequency modulation radio of Armstrong (1933). The invention of new devices is still continuing.

The new principles and new devices appear as answer for the changing conditions of radio communication.

The same developments occur in the field of antenna technology because new antenna systems depend on frequencies and implementation conditions. Below, we discuss which changes in an antenna design are necessary and possible in small-sized systems operating at high frequencies and a wide frequency range.

Recently, communication systems for the population or specialists have been developed. Such systems can operate at frequencies up to several $\mathrm{GHz}$

Corresponding author: Michael Bank, Ph.D., professor, research fields: electrical and communication systems. and must be self-powered and not grounded. Cellular communication is a typical example of such systems.

Here, the use of classical linear antennas such as dipole or monopole antennas, as well as their modifications, is often impossible. The main reasons for this are design features and difficulties in obtaining identical parameters at different frequencies and without grounding.

In this brochure, a new linear-antenna type is proposed. This WBMB (Wide Band MB) antenna allows the operation at different frequencies and wide frequency ranges without grounding. It is based on a single-wire electricity system [1].

All presented results were validated via simulations and measurements using prototype parameters in a chamber laboratory. The monopole antenna is one of the most popular antennas used in communication systems. Its first implementations, introduced by Marconi at the beginning of the 20th century, were at low frequencies and in stationary devices with proper grounding. However, "times are changing", and today various monopole antennas are employed at both low and high frequencies inside small and bigger mobile devices used far from good RF ground systems. It is believed that antennas such as dipole or monopole antennas are resonant devices that operate at a certain frequency. However, many modern transducers operate at several frequencies. For example, 
smartphones operate at two or three frequencies in the range of 0.7-2.2 GHz, and it is expected that this range will be extended to 4 or $5 \mathrm{GHz}$.

Obviously, the development and implementation of wireless communication will continue to grow. In the near future, we will need simple small antennas that operate at several frequencies with different radiation patterns, different polarizations, and in different conditions.

Here, we present the development of a high-performance small wideband antenna that considers all modern, sometimes controversial, requirements. A monopole antenna is a radiator that transmits and receives signals via one wire. Thus, it is an example of SLE (single-line electricity). Its principles and systems are described in Ref. [1]. The MB antenna, a new antenna type, is based on SLE [2-4].

This article does not provide detailed instructions for the development of different MB antennas but introduces simple antennas with new features.

To facilitate the comparisons of results, most simulations and experiments were done at $1 \mathrm{GHz}$ (wavelength $\lambda=30 \mathrm{~cm}$ ).

\section{Grounding Is Zeroing}

According to literature regarding antennas, the height of a monopole antenna should be equal to a quarter of the wavelength, i.e., $\lambda / 4$, or $n * \lambda / 4$, where $n$ is an odd integer. Decreasing or increasing the antenna height leads to deteriorations of the antenna parameters. Further, the signal is fed between the radiator and ground.

However, in this brochure, we show that this is not necessarily true.

As usual, an antenna is described via measurements of its parameters in special chambers. The antenna is not in a transceiver housing and does not experience any external influences. Regarding a real small battery-fed transceiver, the absence of good grounding strongly affects the system.
Consequently, measurements in chambers with ideal grounding do not reflect the quality of antennas under real conditions. The same can be said about the simulation results.

Apparently, each factor must be considered separately. In this study, we investigated the effect of real zeroing in detail.

At high frequencies, the inversion can be realized via a delay line for half period (Fig. 1) or via a half-wavelength-long wire (see figure on the left in Fig. 2).

In the simplest case, a source is connected at one point to a linear radiator and at the other to a nullifier. For maximum gain and efficiency, the radiator length must be $\lambda / 4$, i.e., $7.5 \mathrm{~cm}$ for $f=1 \mathrm{GHz}$.

If a multi-band antenna is required, a multi-ring nullifier should be employed, as shown in Fig. 3.

It is possible to merge several rings into a single wide ring. To illustrate this, we investigated a monopole antenna in which the ground plate is replaced by a wide ring, as shown in Fig. 4. The simulation results at few frequencies confirm that the antenna performance is not deteriorated.

Further, instead of a wide ring, a disk with a perimeter equal to half the wavelength at low frequencies can be used as shown in Fig. 5. We have previously shown that at frequencies above $0.5 \mathrm{GHz}$, the ground plane can be a metal surface of any shape. The perimeter of the surface must be at least half the wavelength of the lowest frequency.

In this case, the metal plate does not radiate as a second dipole metal wire or rod. This was proved for monopoles using a short wire as a nullifier, as shown in Fig. 6. If instead of a ring, a rod of the same height is used as ground, the radiated field decreases by a

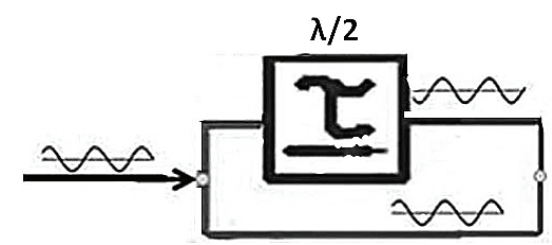

Fig. 1 Nullifier for single-frequency signal. 

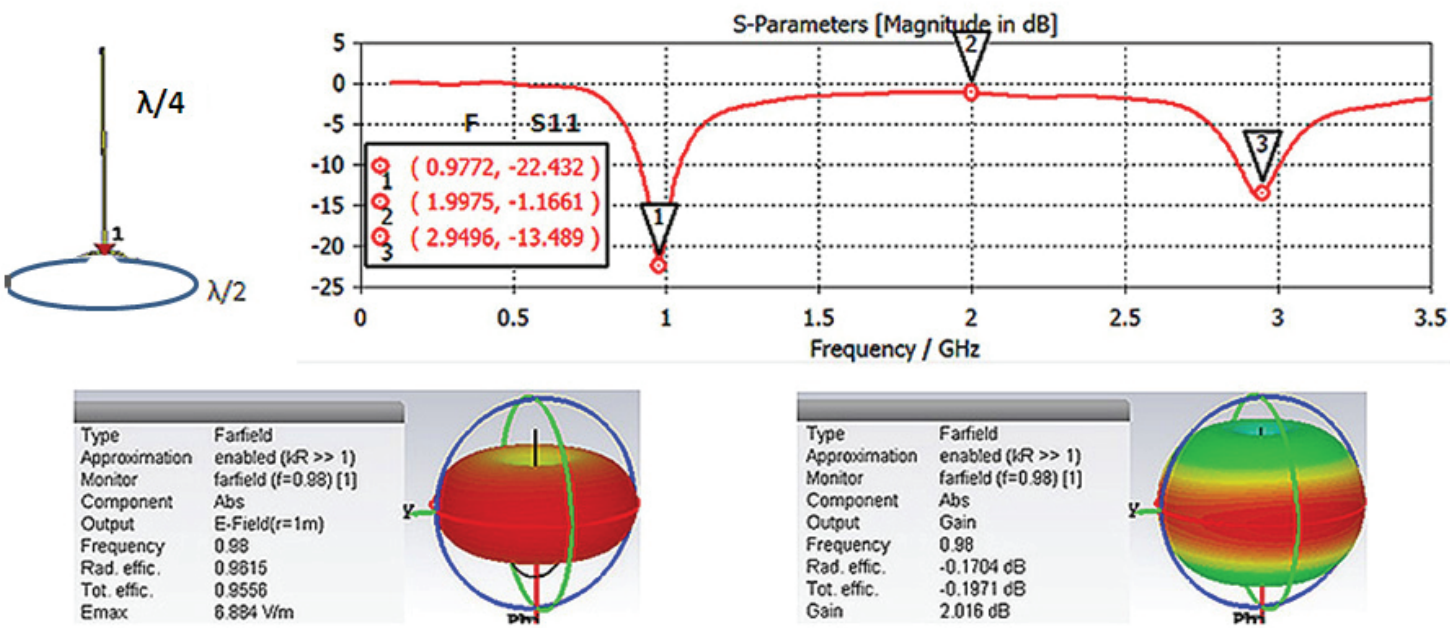

Fig. 2 Monopole antenna with nullifier and simulation results.

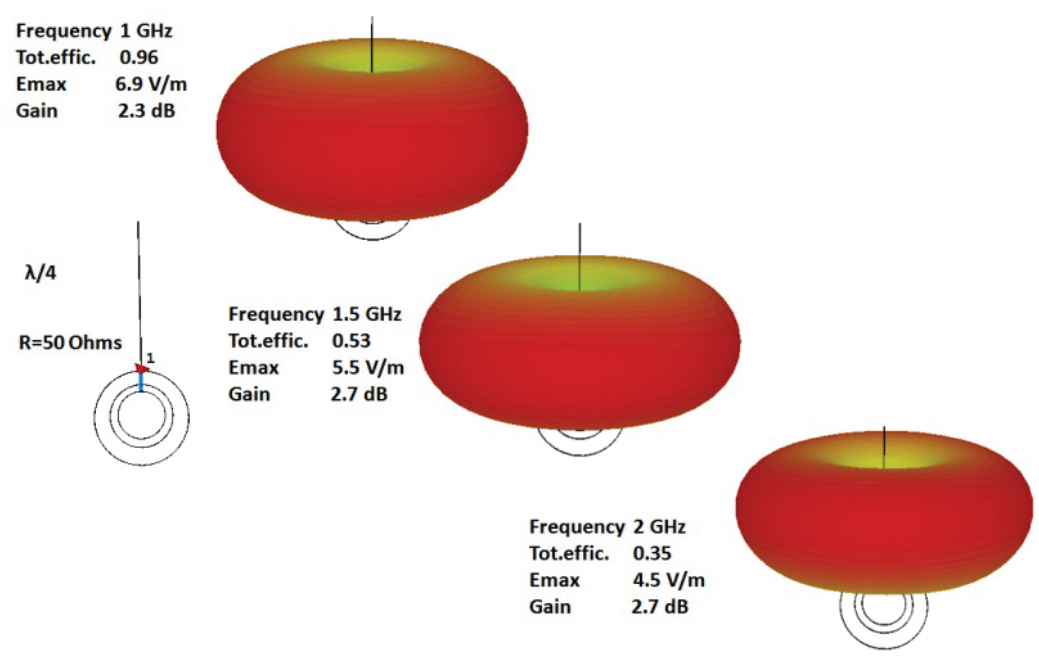

Fig. 3 Monopole antenna working at three frequencies.

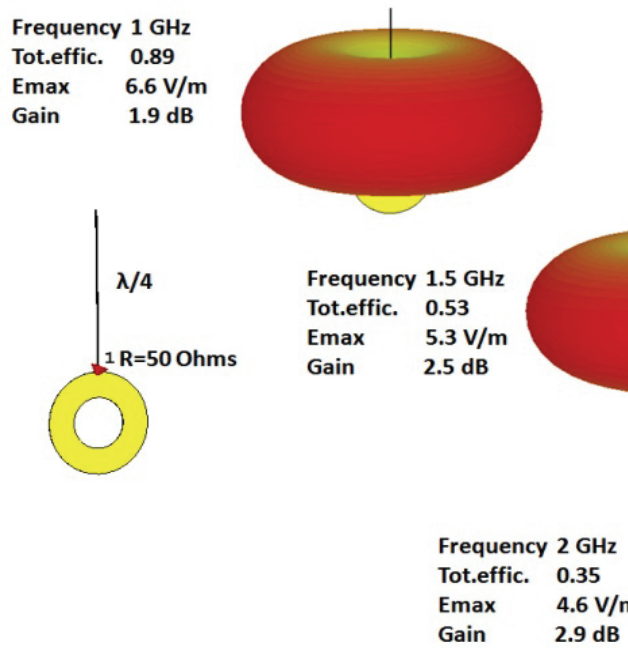

Fig. 4 Monopole antenna with nullifier in the form of a single wide ring.

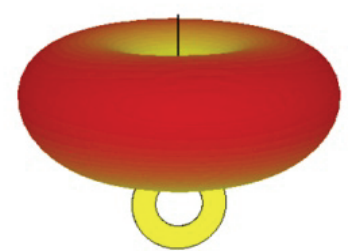




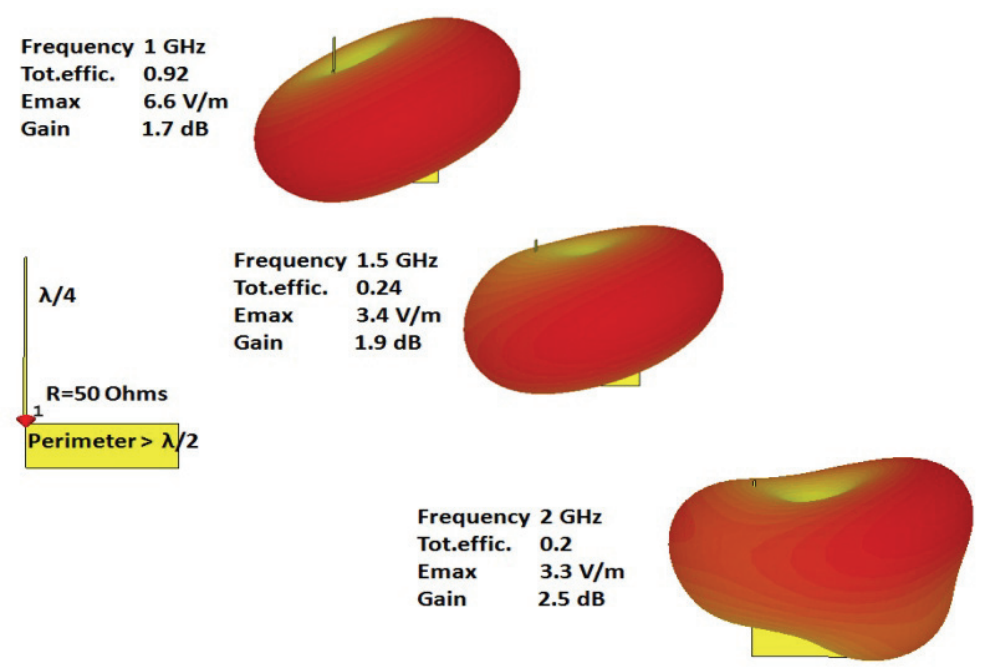

Fig. 5 Monopole antenna with nullifier in the form of a ground plane.
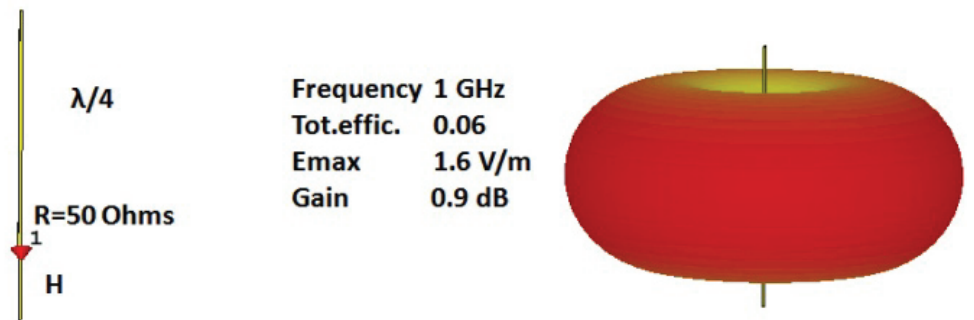

Fig. 6 Monopole with nullifier in the form of a short wire.
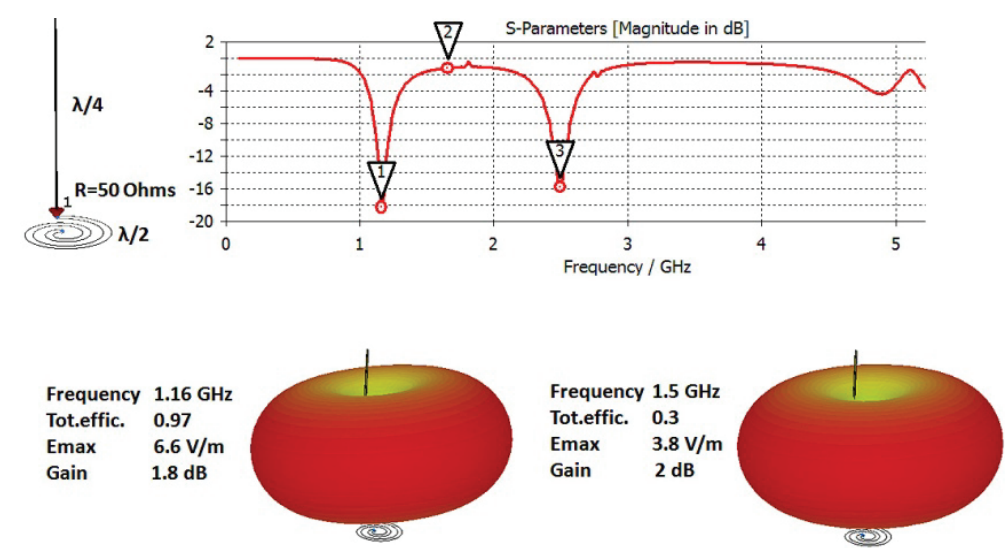

Fig. 7 Monopole antenna with nullifier in the form of a bifilar coil (bottom) and simulations results (graph on upper right).

factor of 10. This proves that not the height but the area/perimeter of the board has an influence on the radiated field.

Further, it is possible to realize nullifiers with bifilar coils. Their special form prevents radiation and hence functions as an ideal nullifier (see Fig. 7).

In mobile devices, a PCB (printed circuit board) or another metallic layer is used for potential zeroing. In this case, the PCB or metallic layer is referred to as the GP (ground plane). However, based on the analysis presented above and the obtained results, good grounding is only achieved if a continuous metallic line of more than half the wavelength of the lowest frequency can be drawn on the PCB.

The hypothesis proposed in Ref. [1] enables us to explain where the current entering the earth disappears and why zeroing occurs. One can tell that grounding works as an aerial consisting of a considerable 
quantity of monopole antennas with lengths much shorter than a quarter wavelength. Such monopole antennas have a very small input resistance and radiate a very small electromagnetic field. If this hypothesis is correct, it is possible to realize a nullifier in the form of a device isolated from the earth and other conductive objects. This nullifier can consist of several small plats that offer paths for the current flow such as in short monopole antennas.

An example of a monopole antenna with this nullifier type is illustrated in Fig. 8.

Fig. 9 presents the simulation results of the monopole antenna in Fig. 8.

The simulation results show that the small nullifier works like a real ground.

In practice, in the case of small transceivers not touching the ground, a PCB, i.e., a GP, is used for zeroing.

Numerous simulations and experiments were carried out to evaluate the work of a PCB as a nullifier. A monopole model without a ground was chosen.

To nullify the potential of one of the source outputs, a PCB, nullifier (Fig. 8), or both were used simultaneously. By changing the height of the monopole antenna, measurements at frequencies corresponding to a quarter wavelengths could be conducted.

The results are the following: The operation with only a nullifier or a combination of a nullifier and a PCB exhibits at all monopole antenna heights a good matching of source and antenna.

Regarding the operation with a PCB only, normal matching was found when the PCB perimeter $(\mathrm{P})$ was approximately a quarter wavelengths. Apparently, in these cases and according to the hypothesis of Ref. [1], the PCB worked as a nullifier.

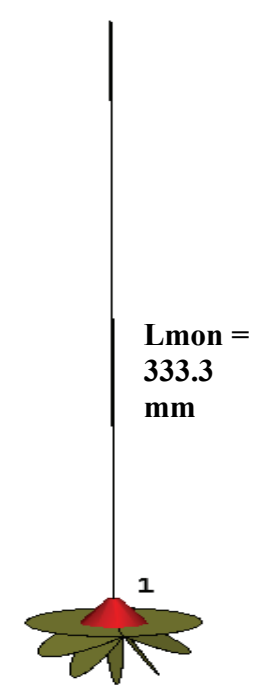

Fig. 8 Monopole antenna with small nullifier

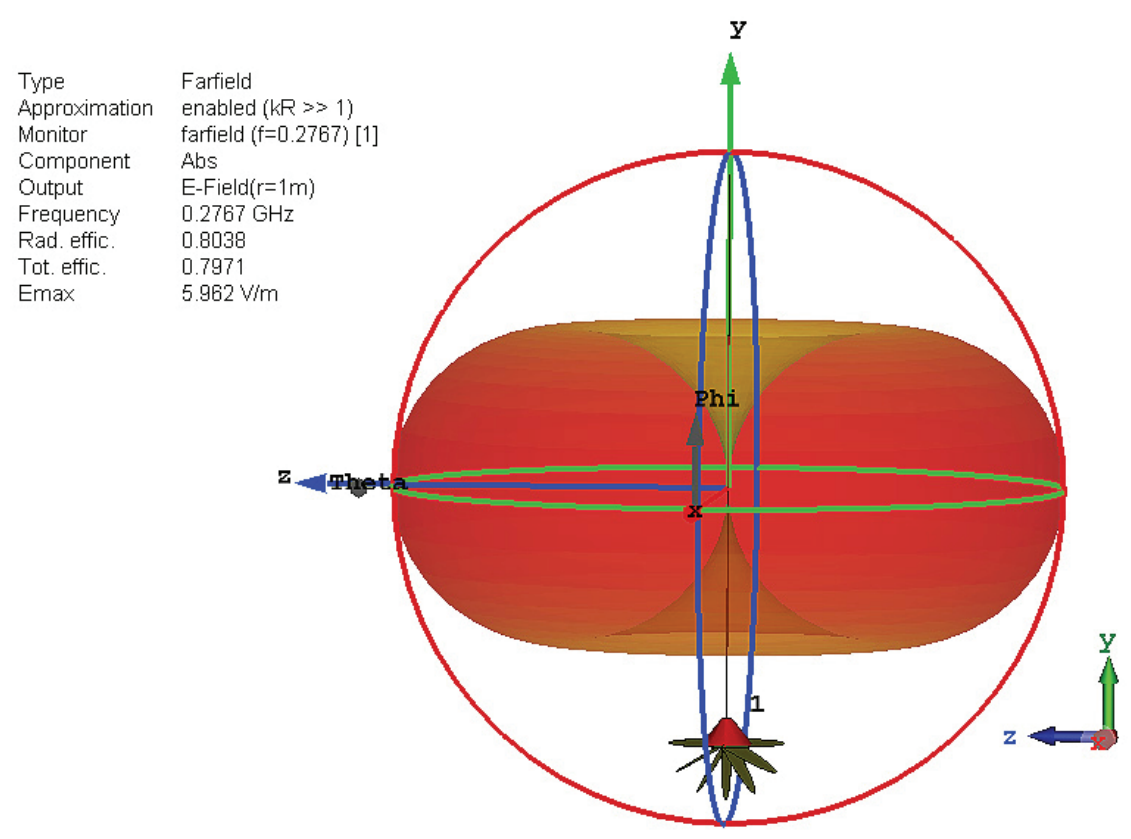

Fig. 9 Simulations result of monopole antenna in Fig. 8. 


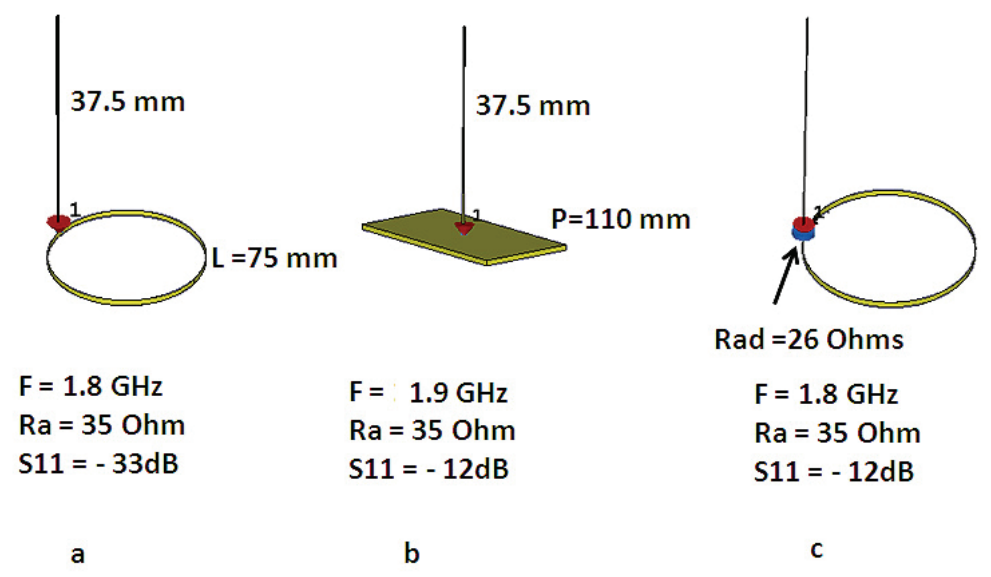

Fig. 10 Estimation of losses caused by nonideality of nullification of real PCB.

The following simulations were done to estimate the losses during the usage of a PCB instead of a nullifier (see Fig. 10).

In Fig. 10a, a monopole antenna with a ring of a length equal to half the wavelength as a nullifier and the simulation results are presented.

In Fig. 10b, the same monopole antenna above a plate (instead of a PCB) with a perimeter of more than half the wavelength is illustrated. In this case, S11 changes from -33 to $-12 \mathrm{~dB}$.

Fig. 10c expresses that the same value, $\mathrm{S} 11=-12$ $\mathrm{dB}$, can be obtained if instead of a nullifier a resistor with $\mathrm{Rad}=26 \mathrm{Ohm}$ is added.

Hence, by using a PCB as a nullifier (as a GP), we deteriorated the antenna efficiency by approximately $26 /(26+35) \times 100 \%=43 \%$.

\section{Wideband Monopole}

It is usually assumed that a monopole antenna exhibits only the best performance if its height is equal to an odd multiple of a quarter wavelength. Further, a half-wave monopole antenna does not radiate because the radiation from the quarter-wave segments cancel each other. This is illustrated by the current distribution along a quarter-wave and half-wave monopole antenna in Fig. 11.

The far field of a $\lambda / 2$ monopole antenna is smaller than that of a $\lambda / 4$ one.

However, considering that monopole and dipole antennas are devices that convert electrical current into electromagnetic radiation, no constraint on their operating frequency range should exist.

Based on the antenna theory and principles of the single-line method for the transmission of energy and information, we can assert the following:

- Normal antennas are passive devices and cannot amplify the signal power.

- If the ohmic resistance of an antenna is smaller than its radiation resistance, the antenna loses only a small amount of the input signal power.

- The total efficiency of an antenna decreases owing to poor matching between source resistance and antenna input resistance.

- By manipulating the input signal's phase, it is possible to avoid that the radiation from the antenna segments is cancelled.

- Good matching and phase shift of the input signal (by compensating the reactive resistance of the antenna) cause improved parameters at all frequencies.

In order to validate this principle, we simulated a half-wave monopole with high active and reactive radiation resistances. Fig. 12 shows that a tuned monopole (i.e., a quarter-wave monopole antenna) operating at $1 \mathrm{GHz}$ exhibits an active resistance of 200 $\Omega$ at $2 \mathrm{GHz}$ (instead of $50 \Omega$ at $1 \mathrm{GHz}$ ) and a large negative reactive resistance.

In conclusion, two main reasons exist for the 


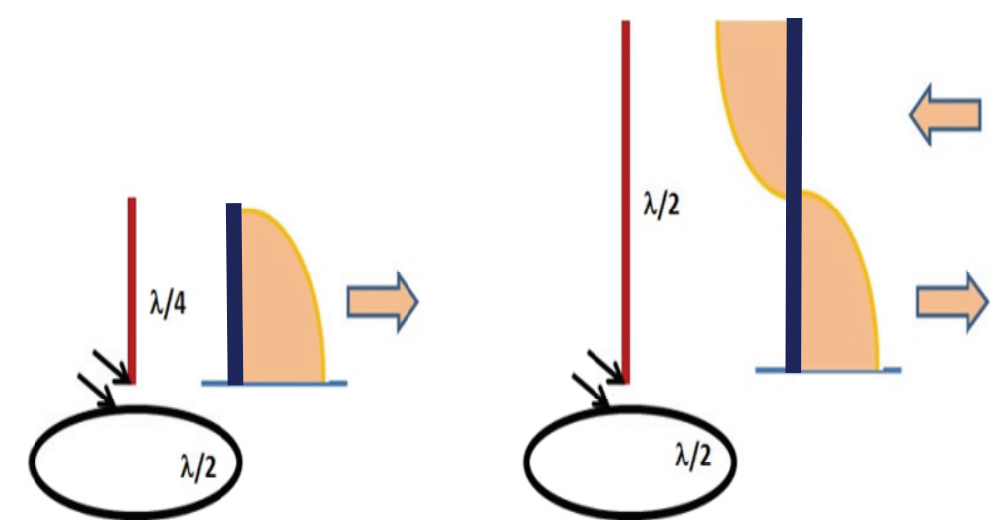

Fig. 11 Current distribution in $\lambda / 4$ and $\lambda / 2$ monopole antennas.
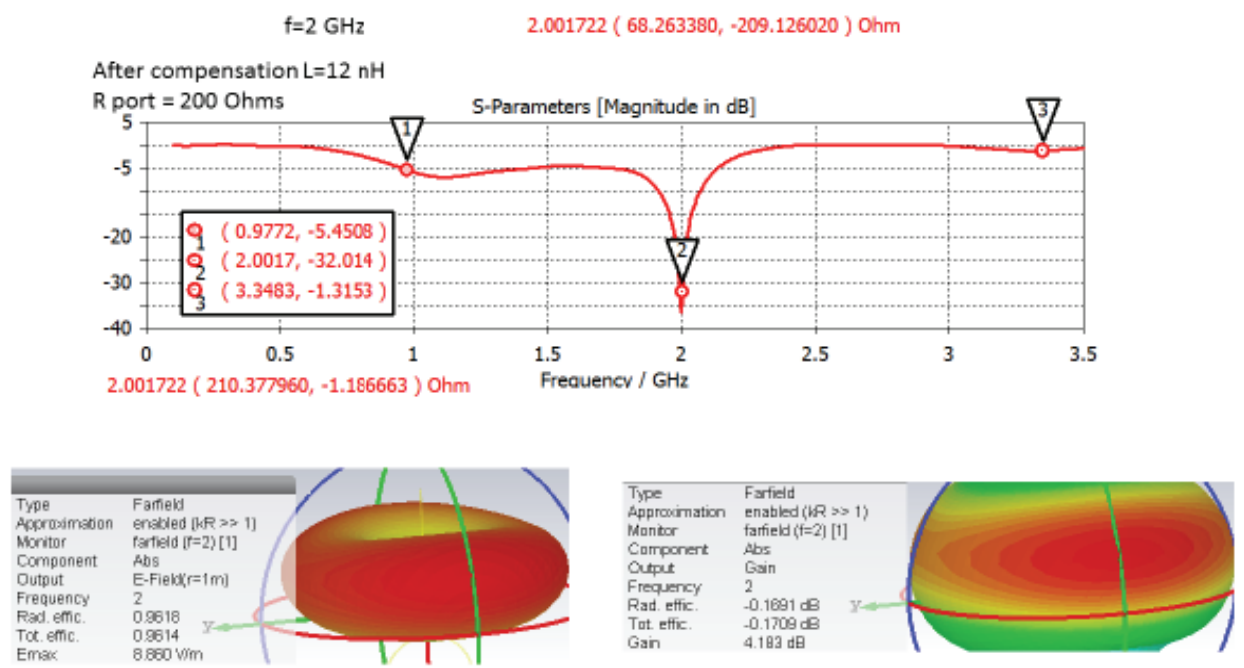

Fig. 12 Simulation results of $\lambda / 2$ monopole antenna with active resistance matching and reactive resistance compensation.

deterioration of the parameters of a monopole antenna with a change in its length from a quarter of the wavelength. These reasons are the varied (active) radiation resistance and the appearance of a reactive resistance.

Subsequently, a monopole antenna can be modified to work at all frequencies. However, if one uses a source resistance of $200 \Omega$ and adds a compensating inductor of $12 \mathrm{nH}$, the antennas operating at $2 \mathrm{GHz}$ work as well as at $1 \mathrm{GHz}$.

This is confirmed by the simulation results presented in Fig. 12.

The presented analysis and results show that it is impossible to design a wideband monopole antenna by adding compensating reactive elements. This is due to the fact that the magnitude and sign of the reactive radiation resistance changes with the frequency. Further, it is impossible to choose the medial reactive resistance to enable compensations along the complete bandwidth. In the next section, we explain how this problem can be solved.

\section{MB Antenna}

Recently, a new monopole antenna type, the so-called MB antenna, has been proposed in Ref. [2]. The main goal was to realize small antennas with only one radiating element and without any GP, which exhibit high performances like tuned dipoles. Fig. 13 shows how a conventional dipole is transformed into an MB antenna. 


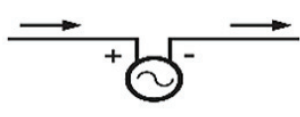

a

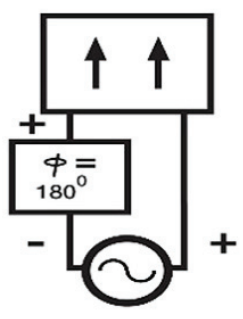

c
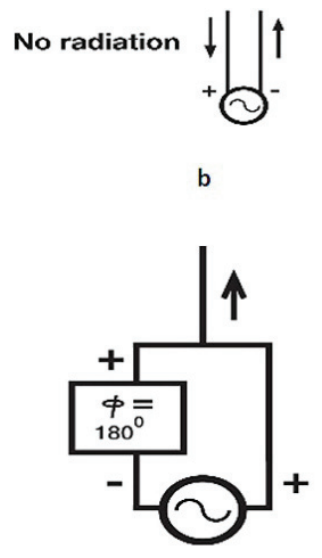

d

Fig. 13 MB antenna structures.

As shown in Fig. 13a, the arms of a conventional dipole antenna are collinearly aligned so that the currents in both dipole arms flow in the same direction, thereby leading to a high radiation efficiency. If the arms are positioned in parallel as in Fig. 13b, e.g., owing to space constraints, the currents flow in opposite directions and the resulting radiated fields cancel each other. Currents with identical directions and hence efficient radiation can be obtained by introducing a phase shift of $180^{\circ}$ (an inverter) to one of the source leads, as depicted in Fig. 13c, where the radiating element is a single wire. This is the main idea behind MBA. Fig. 13d shows another MBA version in which the PCB of a mobile device is used as radiating element, thereby avoiding a separate antenna. Thus, we realized an antenna with one radiating element and without a ground. More details about MBA can be found in Refs. [3-6].

A $180^{\circ}$ phase shifter (inverter) implemented via a delay line designed for a frequency $\mathrm{F}$ provides the same phase shift at frequencies of, e.g., $3 \mathrm{~F}$ and $5 \mathrm{~F}$.

The additional important advantage of an $\mathrm{MB}$ antenna is that it not only works at odd harmonics (3F, $5 \mathrm{~F}, \ldots)$ of the main frequency $\mathrm{F}$, but also at even harmonics $(2 \mathrm{~F}, 4 \mathrm{~F}, \ldots)$. This is achieved by the fact that the delay line (inverter) at odd frequencies F, 3F, $5 \mathrm{~F}$... can operate as an antenna at even frequencies $2 \mathrm{~F}, 4 \mathrm{~F}, \ldots$. Hence, if the delay line has the form of an

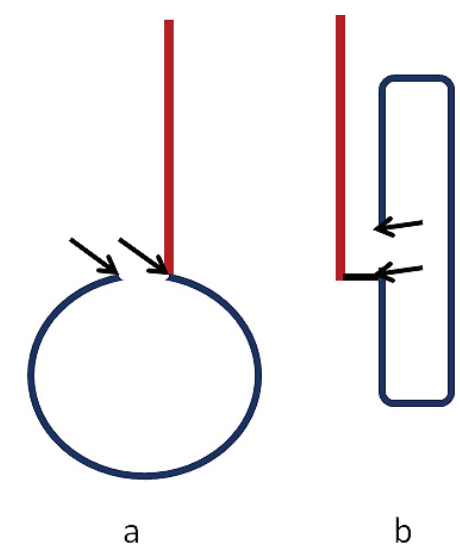

Fig. 14 Two examples of WBMB antenna with (a) ring and (b) Pistohlkors antenna.

antenna, a wideband antenna is realized.

For this purpose, a delay line with a special form can be chosen, the electric length of which is equal to half the wavelength at frequency F. The idea is to use a delay line that acts as an antenna at frequency $2 \mathrm{~F}$. Examples of these antennas are a loop (magnetic antenna) or folded dipole (electric antenna), shown in Figs. 14a and 14b, respectively. The folded dipole (electric antenna) was proposed by Pistohlkors in 1936 (patent: RU 2192079C2).

In both cases, the perimeter of the second radiator must be equal to one wavelength at $2 \mathrm{~F}$, and an integer multiple of this wavelength at even harmonics $4 \mathrm{~F}$, $6 \mathrm{~F}, . .$. Hence, an antenna consisting of two elements is obtained. The two elements radiate alternately with increasing frequency.

Below, we illustrate that an MB antenna can have many different implementations: e.g., a wideband feed for parabolic antennas with circular polarization, transparent antennas, or textile antennas.

\section{MB Antenna with Ring and Its Features}

As stated above, a monopole antenna can be implemented with a nullifier instead of a ground. The nullifier can be a half-wavelength circular line. This monopole antenna differs from an MB antenna only in the connection to the source (see Fig. 15).

Fig. 16 shows the simulation results for the antennas of Fig. 15. The main difference between 


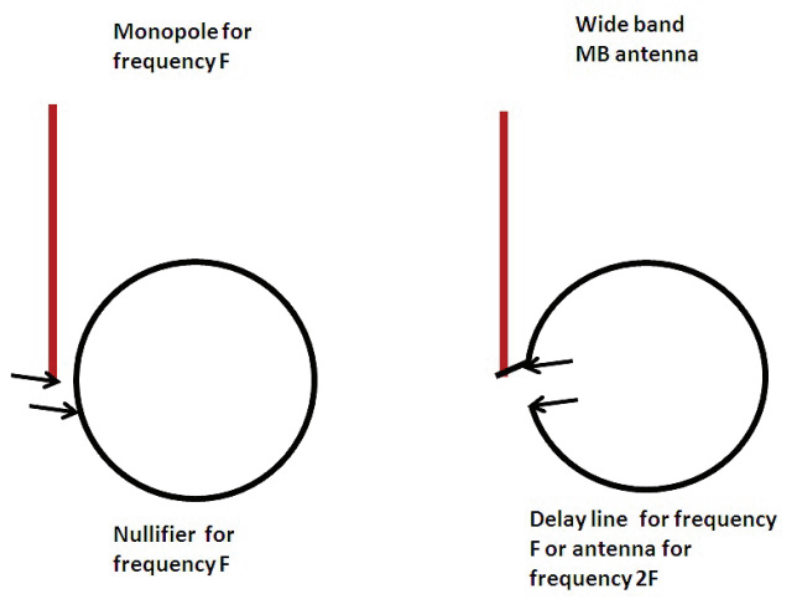

Fig. 15 Monopole and MB antenna with equal delay line. these antennas is that the monopole antenna has good parameters at 1 and $3 \mathrm{GHz}$ but the $\mathrm{MB}$ antenna can also operate at $2 \mathrm{GHz}$.

Hence, an MB antenna works at all frequencies. However, the best parameters are determined via frequencies that correspond to the minima of S11.

If good parameters at another frequency are necessary, two solutions exist: shift the minima of S11 or add reactive components to cancel the reactive part of the antenna impedance. To shift the S11 minima to the desired frequencies, one should change the length of the delay line. Fig. 17 shows shifts in the S11 curve
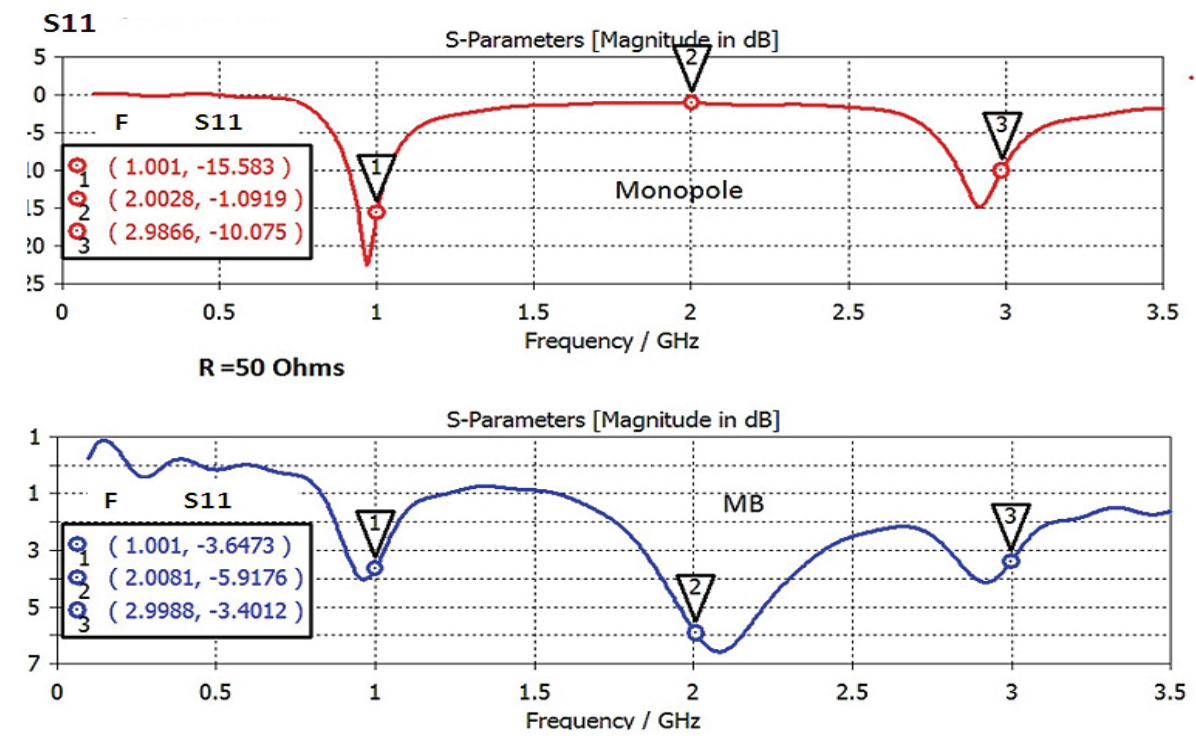

Fig. 16 Simulation results of monopole and MB antenna with equal delay line.

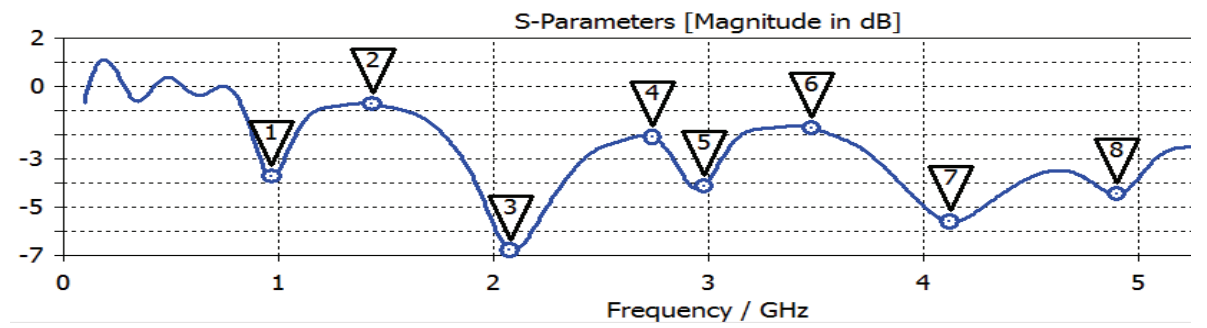

(a)

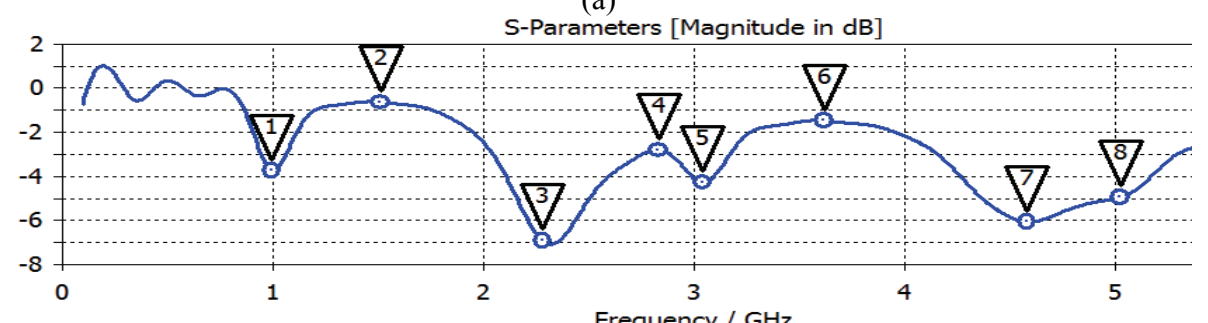

(b)

Fig. 17 Delay line is decreased by $10 \%$ in case $b$. 


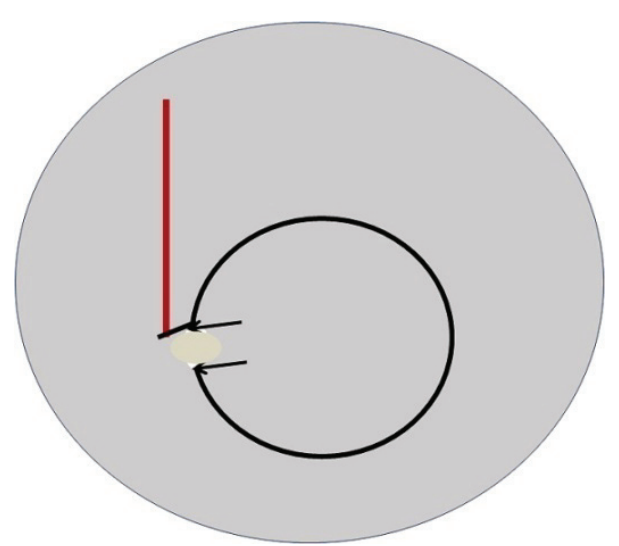

Fig. 18 WBMB antenna with reflector.

as a result of a $10 \%$ decreased delay line.

However, in certain circumstances, e.g., if a high-gain directional antenna is required, this can be an advantage. Such an antenna type can be obtained by integrating a reflector in parallel to the antenna, as shown in Fig. 18.

Fig. 19 presents the simulation results of a parabolic reflector (like those used in satellite communication systems).

By using a reflector, the gain could be increased by 6-10 dB and higher.

\section{Matching between Source Resistance and Antenna Impedance}

The delay line length is designed to achieve the desired spectral response for an antenna. However, if after its optimization the antenna parameters are not satisfactory, compensation components for the reactive resistance can be used.
Let us consider the challenging task of connecting a single wideband MB (WBMB) antenna to several sources with different frequencies. The following problems appear:

First, the sources must not influence each other. Second, each source can have a different real resistance. Third, each source can have a different reactive resistance.

All these problems can be solved using the scheme in Fig. 20 [8].

Let us assume $n$ sources with different frequency bands at which the WBMB antenna has different active and reactive resistances. We assume that the sources are balanced. To harmonize all sources and the WBMB antenna, it is possible to apply a matching reactance $X$ to each source. In this scheme, band pass filters are employed and no adder is necessary. The filter acts simultaneously as a transformer and Balun. Therefore, it is convenient to include a TDRC (transformed double-resonant circuit) in the band pass filters (see Fig. 21).

The circuits in Fig. 19 completely compensate the reactive impedance of the antenna at all desired frequencies. The simulations results in Fig. 22 confirm the effectiveness of the method. The MB antenna with a ring has one disadvantage. Its radiation pattern is not unidirectional at even frequencies because magnetic antennas in the form of loops do not radiate in the loop plane. One example of a radiation pattern is shown in Fig. 22.

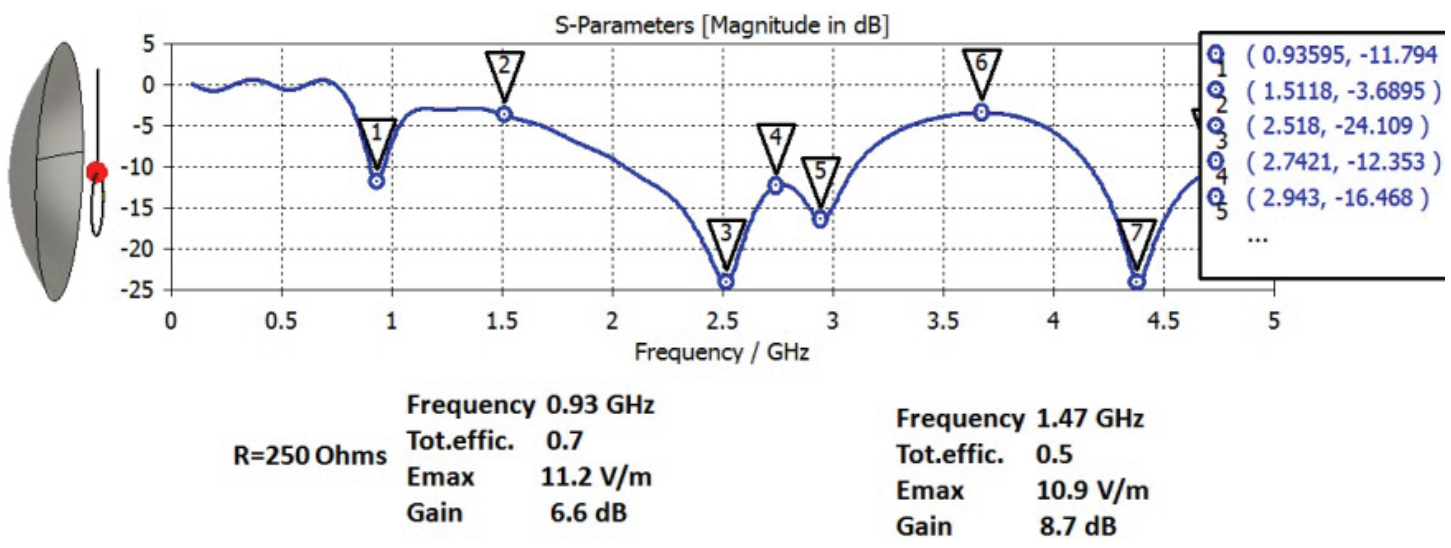

Fig. 19 Simulation results of WBMB antenna with reflector. 


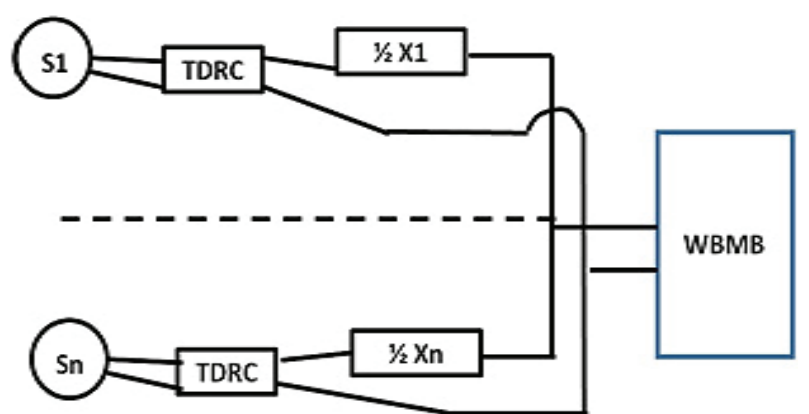

Fig. 20 WBMB antenna with different sources.

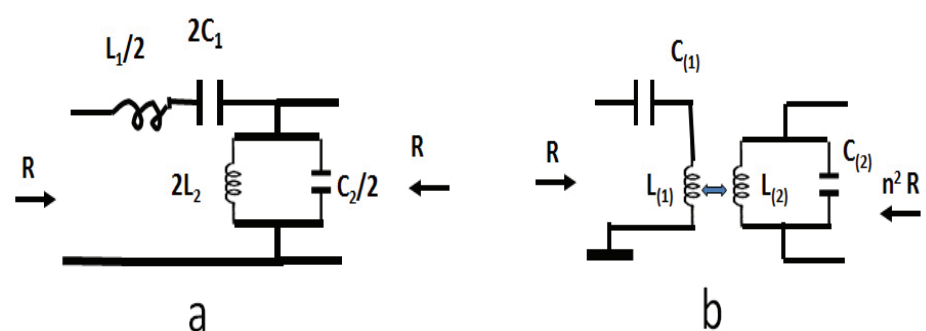

Fig. 21 Transition from (a) conventional band pass filter to (b) transformer band pass filter.

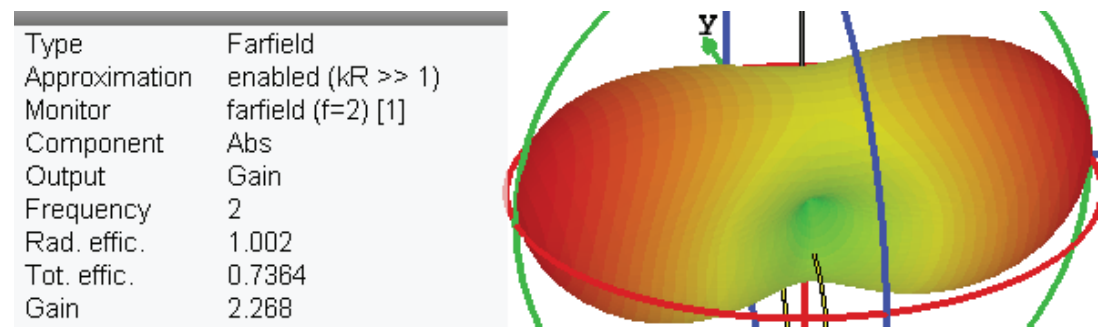

Fig. 22 Radiation pattern of WBMB antenna at frequency $2 \mathrm{~F}=2 \mathrm{GHz}$.

\section{MB Antenna with Folded Dipole Antenna}

The folded dipole or Pistohlkors antenna (Fig. 23) can be used in an MB antenna where it acts as a delay line at odd harmonics of the fundamental frequency and as a radiator at even harmonics.

Fig. 24 shows the respective simulation results at the basic frequency $\mathrm{F}$. The source resistance is 50 Ohm.

The basic parameters at different frequencies are presented in Fig. 25. Evidently, the MB antenna with a folded dipole has omnidirectional radiation patterns.

Regarding wideband antennas, the choice of the source resistance is generally a complicated problem because a wideband antenna has different active radiation resistances at different frequencies.

It is possible to choose individual source resistances at all frequencies according to the scheme in Fig. 20.
However, its usage is complicated.

It is more convenient to use one source resistance at all frequencies. We consider two cases.

Good parameters can be obtained using a source resistance equal to the average value of the radiation resistance along the operating bandwidth. However,

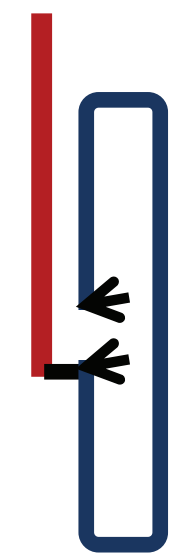

Fig. 23 MB antenna with monopole and folded dipole. 


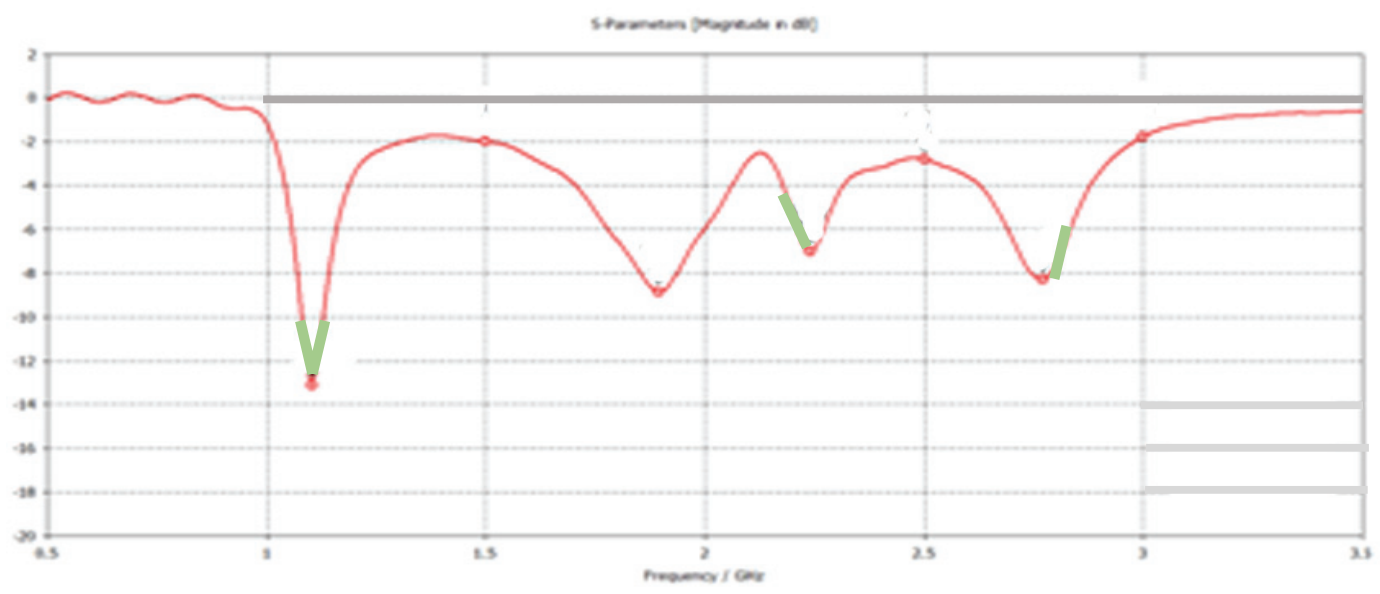

Fig. 24 Simulation results of S11 parameters in MB antenna with folded dipole.
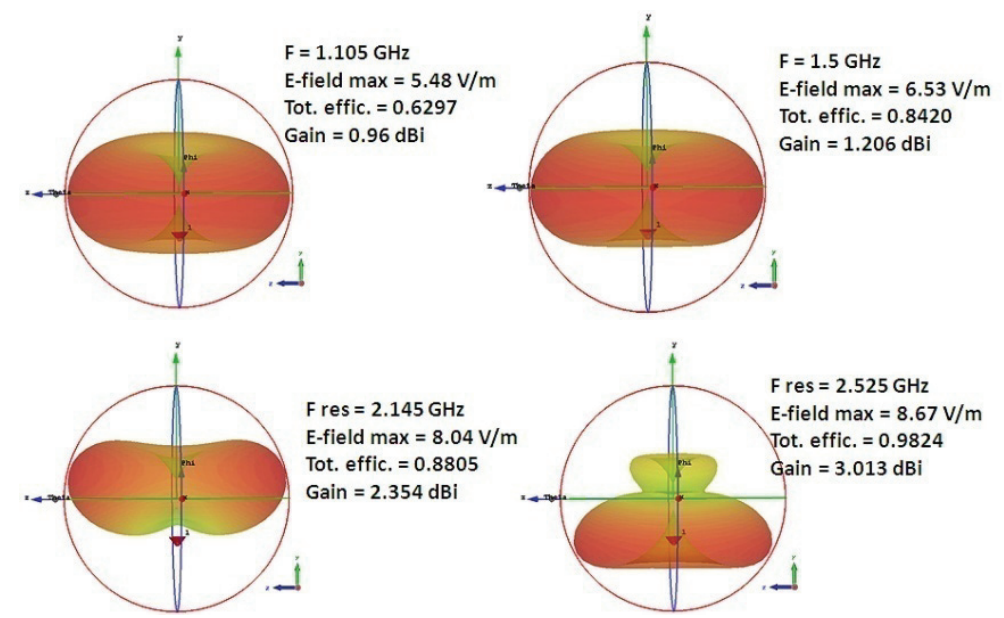

Fig. 25 Radiation patterns of MB antenna.

an unavailable high-frequency wideband transformer is necessary.

Commonly, a source resistance of $50 \mathrm{Ohm}$ is used. Its usage is simple and profitable owing to the coaxial cables with a resistance of $50 \mathrm{Ohm}$ and their connectors. To check the difference between the middle radiation resistance of $300 \mathrm{Ohm}$ and a resistance of $50 \mathrm{Ohm}$, several simulations were conducted. Some simulation results are listed in Fig. 26.

These results show that the antenna parameters at 50 and $300 \mathrm{Ohm}$ do not differ much. The resulting benefit is significant.

An MB antenna is as an electrically small antenna. As mentioned in Section 4, in many cases, the use of shortened antennas is also possible. However, these antennas have a reactive radiation resistance and reduced active component. Compensating these disadvantages is possible but not always feasible. Regarding MB antennas with folded dipoles, the antenna height can be decreased without noticeably

\begin{tabular}{|c|c|c|c|}
\hline $\begin{array}{c}\mathrm{F} \\
\mathrm{GHz}\end{array}$ & $\begin{array}{c}\text { Source } \\
\text { Impedance } \\
\text { Ohms }\end{array}$ & $\begin{array}{c}\text { Gain } \\
\mathrm{dBi}\end{array}$ & $\begin{array}{c}\text { E-field } \\
\mathrm{V} / \mathrm{m}\end{array}$ \\
\hline 1.105 & 50 & 0.78 & 6.54 \\
\hline 1.5 & 300 & 0.96 & 5.48 \\
\hline & 50 & 1.217 & 4.29 \\
\hline$\approx 2.0$ & 300 & 1.206 & 6.53 \\
\hline & 50 & 1.649 & 6.91 \\
\hline \multirow{2}{*}{$\approx 3.5$} & 300 & 2.354 & 8.04 \\
\hline & 50 & 2.965 & 5.96 \\
\hline & 300 & 3.013 & 8.67 \\
\hline & 50 & 2.662 & 7.69 \\
\hline
\end{tabular}

Fig. 26 Simulation results for different resistances. 

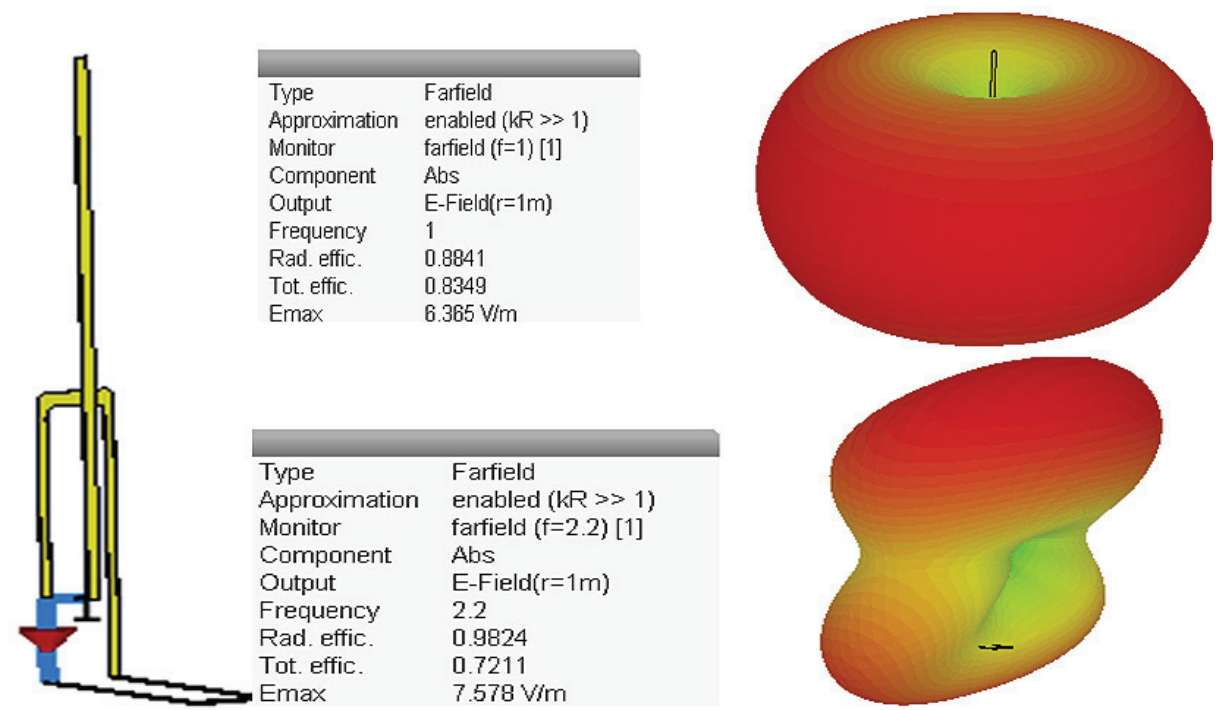

Fig. 27 Bent MB antenna and simulation results.

deteriorating the parameters. This method and the respective simulation results are presented in Fig. 27. This method exhibits satisfactory parameters.

\section{WBMB Antenna Working at All Frequencies in Its Frequency Band Using Compensation}

Any linear antenna can work at several frequencies if the length of the antenna is equal to an odd multiple of a quarter wavelength. Below, we call these frequencies "resonant frequencies".

However, if an electrical current exists in the wire, an electromagnetic field exists around the wire. We can assume that these antennas have no optimal parameters at resonant frequencies owing to large reactive resistances. Therefore, if the reactive resistances are compensated, the antenna parameters at all frequencies can be significantly improved.

Let us consider the construction of an antenna with reactance compensation. The antenna should operate from a symmetrical source with a resistance of $50 \Omega$ in the range of $0.85-5 \mathrm{GHz}$.

To meet all the requirements, a folded monopole and folded dipole (Pistohlkors antennas) have to be installed. The selected antenna design is shown in Fig. 28.
The simulation results for $4 \mathrm{GHz}$ show that an equivalent antenna circuit at $4 \mathrm{GHz}$ presents a serial connection of the active resistance and an inductive resistance of $110 \mathrm{Ohm}$.

Therefore, a capacitor of $0.33 \mathrm{pF}$ must be included for compensation between the source and antenna. Fig. 29 shows the $\mathrm{S} 11$ parameters at $4 \mathrm{GHz}$ before and after compensation as shown in Fig. 30.

If it is necessary to work at several frequencies, the compensation elements must be divided. In this case, it is possible to include a series resonant circuit in

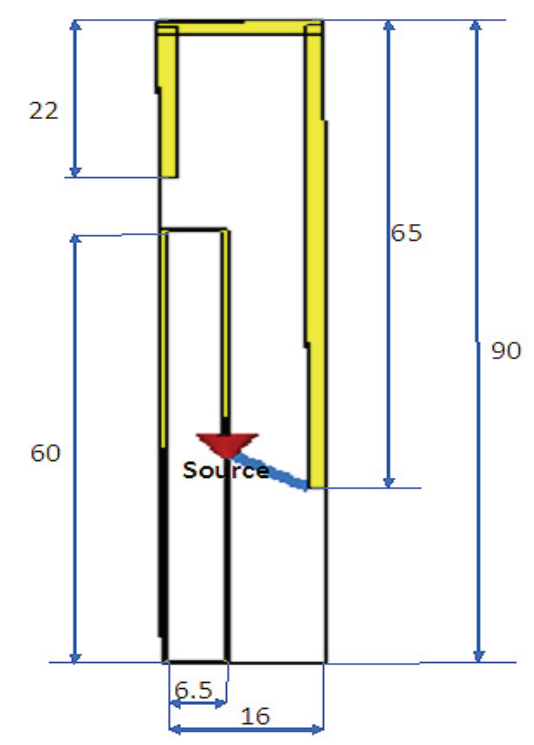

Fig. 28 Widen small MB antenna example. 


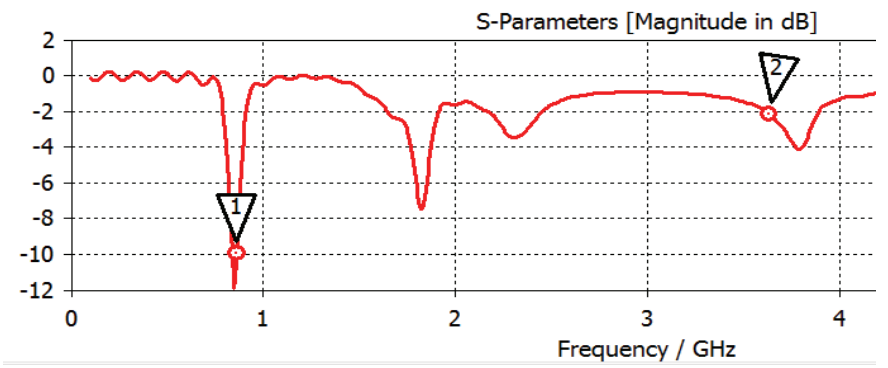

(a)

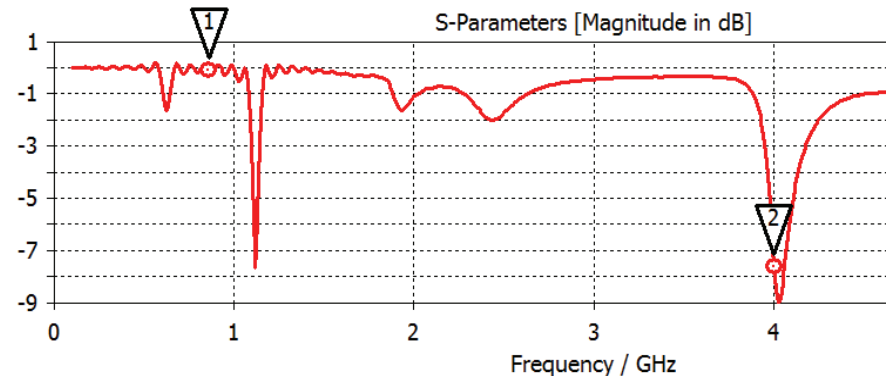

(b)

Fig. 29 S11 parameters of antenna in Fig. 28.

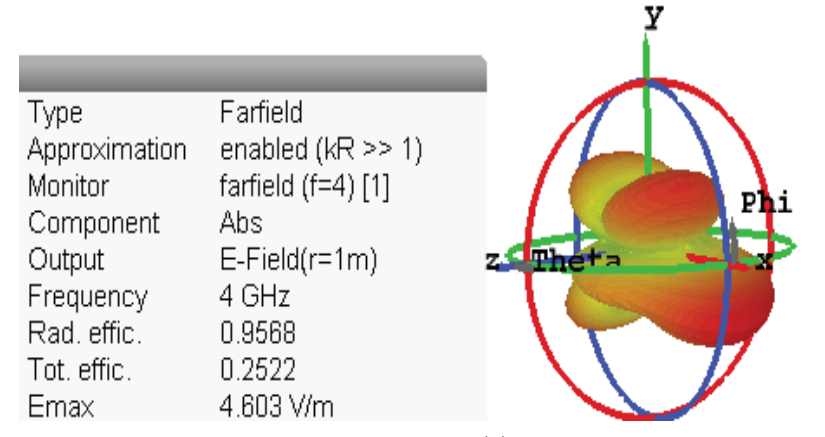

(a)

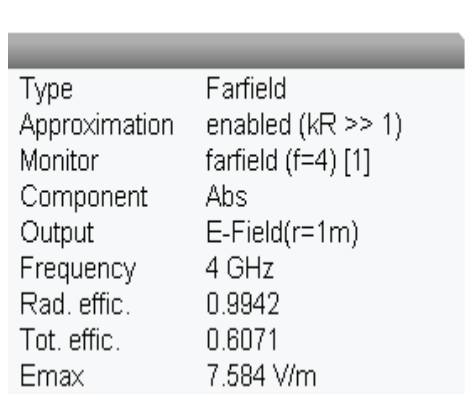

(b)

Fig. 30 Results of antenna in Fig. 28; (a) before and (b) after compensation at $4 \mathrm{GHz}$.

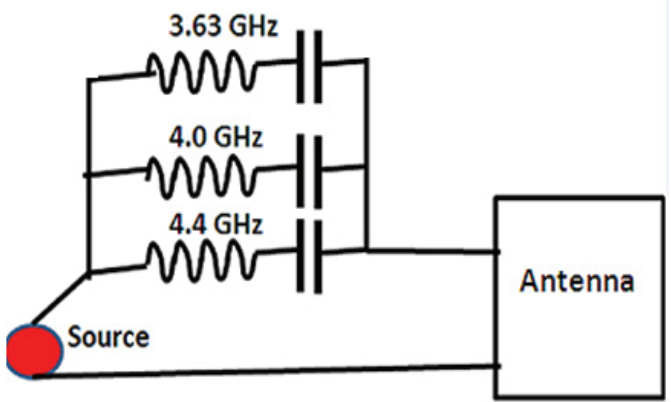

Fig. 31 Reactive resistance compensation at three frequencies.

series with the compensation element, and reactive elements of the same sign can be combined.
All compensation and circuit elements for all frequencies must be connected to operate simultaneously at all frequencies. The circuits are connected in parallel with each other (for three frequencies) and in series with the source and antenna, as shown in Fig. 31.

Fig. 32 presents the S11 parameters after compensations at frequencies 2,3 , and 4 .

The simulation results are listed in Table 1.

In this table, three values are indicated for each frequency: "non-compensated", "compensated", and "compensated and with resonant circuit". 


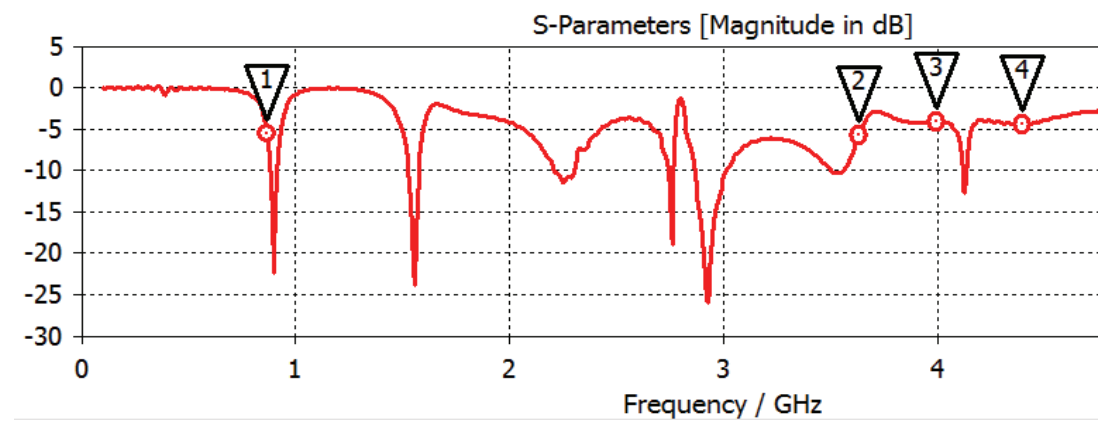

Fig. 32 S11 parameters of antenna in Fig. 26 after compensation.

Table 1 Compensation elements values and results in antenna in Fig. 31.

\begin{tabular}{|c|c|c|c|c|c|c|c|c|}
\hline $\begin{array}{l}f, \\
\mathrm{GHz}\end{array}$ & $\begin{array}{l}\text { Model } \\
\text { number }\end{array}$ & $\mathrm{S} 11, \mathrm{~dB}$ & E-max, v/m & Total effic. & Gain, dB & $\mathrm{Ra}, \mathrm{Ohms}$ & $\mathrm{X}, \mathrm{Ohms}$ & $\begin{array}{l}\text { Compensation and } \\
\text { oscillatory circuit elements }\end{array}$ \\
\hline & 1 & -10 & 5.9 & 0.73 & 1.1 & 80 & 24 & \\
\hline \multirow[t]{3}{*}{0.86} & 2 & -3 & 4.7 & 0.47 & 2.2 & 94 & 136 & \\
\hline & 3 & -4 & 5.4 & 0.6 & 1.9 & 74 & 96 & \\
\hline & 1 & -2 & 5.9 & 0.37 & 4.9 & 20 & -76 & - \\
\hline \multirow[t]{3}{*}{3.63} & 2 & -7 & 7.7 & 0.79 & 2.7 & 18 & 4 & $\mathrm{~L}=3.2 \mathrm{nH}$ \\
\hline & 3 & -18 & 11 & 0.95 & 3.7 & 18 & 34 & $\mathrm{~L}=6.5 \mathrm{nH}, \mathrm{C}=0.6 \mathrm{pF}$ \\
\hline & 1 & -1 & 4.6 & 0.25 & 5.6 & 24 & 112 & - \\
\hline \multirow[t]{3}{*}{4} & 2 & -3 & 7.1 & 0.54 & 5.1 & 261 & -44 & $\mathrm{C}=0.33 \mathrm{pF}$ \\
\hline & 3 & -4 & 6.5 & 0.55 & 4.5 & 193 & -55 & $\mathrm{~L}=4 \mathrm{nH}, \mathrm{C}=0.2 \mathrm{pF}$ \\
\hline & 1 & -1 & 3.9 & 0.17 & 4.7 & 307 & 475 & - \\
\hline \multirow[t]{2}{*}{4.4} & 2 & -5 & 8 & 0.71 & 5.4 & 116 & 73 & $\mathrm{C}=0.054 \mathrm{pF}$ \\
\hline & 3 & -4 & 7.1 & 0.63 & 4.5 & 152 & 82 & $\mathrm{~L}=0.16 \mathrm{nH}, \mathrm{C}=0.03 \mathrm{pF}$ \\
\hline
\end{tabular}

All compensation elements can be combined if it is possible to work with one source resistance. If the active resistance of the antenna is different at different frequencies, several transformers are necessary or the solution presented in Figs. 20 and 21 must be employed.

\section{MB Antenna with Circular Polarization}

WBMB antennas can be implemented as a wideband feed of parabolic satellite antennas. However, they usually must have circular polarization. Antennas with circular polarization are often made of different spiral antennas.

Circular polarization can be achieved using two sources with orthogonal vectors. Therefore, it is possible to use two WBMB antennas with different sequences, E-H-E-H and H-E-H-E ("E" for electric field and " $\mathrm{H}$ " for magnetic field) for different frequencies. By combining these antennas, an antenna with circular polarization is obtained. For this purpose, the base frequency of the first antenna is $\mathrm{F}$ and the frequency of the second antenna is $2 \mathrm{~F}$.

We chose two WBMB antennas with different loop antenna parameters to realize an antenna with circular polarization (see Fig. 33).

In Fig. 34, the S11 simulation results for each antenna are presented.

In Fig. 35, the simulations results of the combined antennas are presented.

To examine the existing circular polarization, we needed to determine the axial ratio. The axial ratio is the ratio of the orthogonal components of an

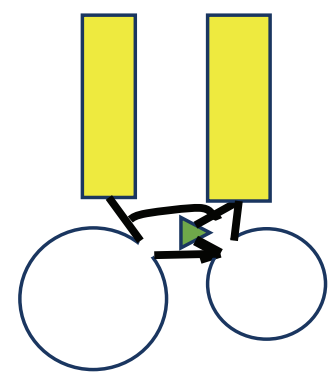

Fig. 33 Two MB antennas for circular polarization. 


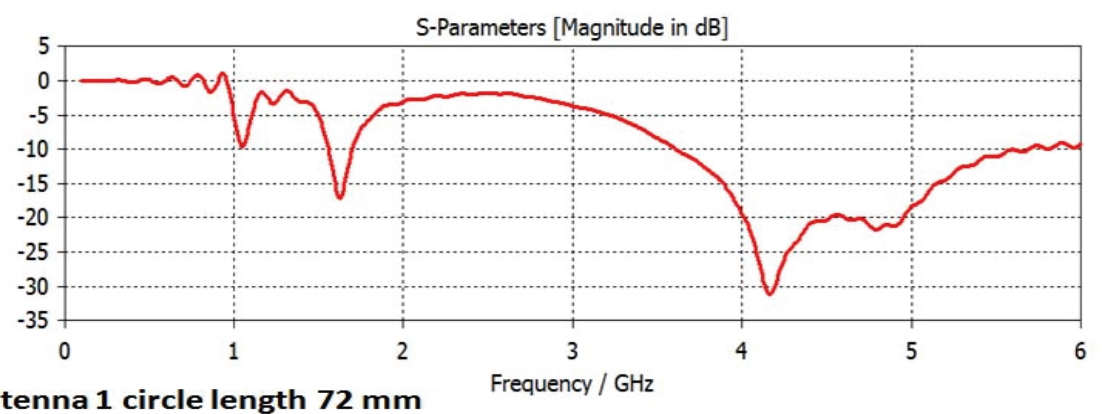

Antenna 1 circle length $72 \mathrm{~mm}$

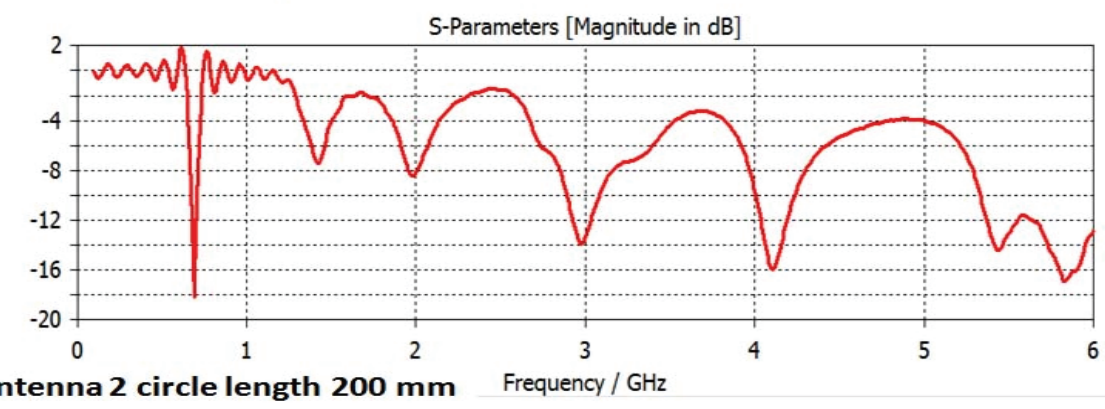

Fig. 34 S11 parameters of each antenna.

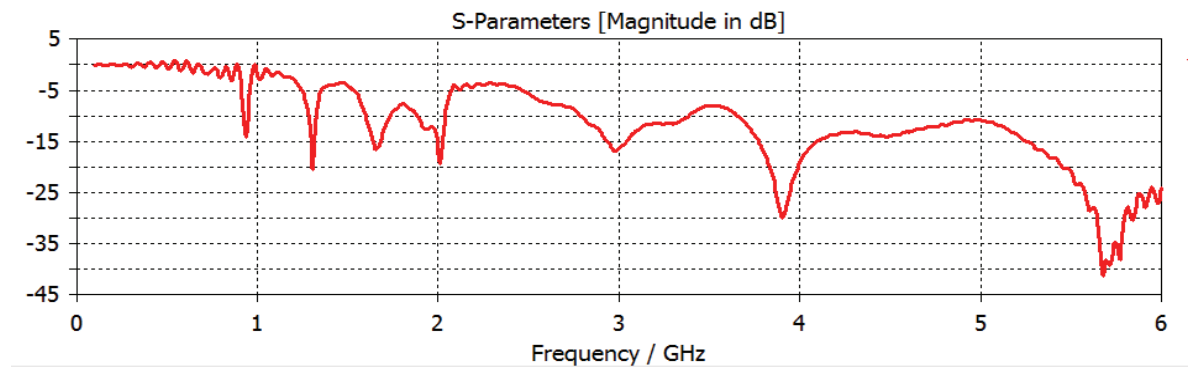

Fig. 35 S11 parameters in combined antennas.

electromagnetic field. A circularly polarized field is made of two orthogonal components of equal amplitude (and $90^{\circ}$ out of phase). If the components on the $\mathrm{X}$ and $\mathrm{Y}$ axes have the same level, a circular polarization can potentially be realized. The simulation results of the axial ratio at $3.9 \mathrm{GHz}$ are presented in Fig. 36.

Fig. 37 presents the components on the $\mathrm{X}$ and $\mathrm{Y}$ axes at $3.9 \mathrm{GHz}$ separately.

The simulation results confirm that a circularly polarized antenna can be realized with two WBMB antennas.

\section{MB Antenna with Wide Radiator}

One possible application of $\mathrm{MB}$ antennas is an antenna with a wide radiator. For example, a transparent antenna instead of glass could be used as a car window. The radiator is covered with transparent conductive films, e.g., ITO (indium tin oxide) and FTO (fluorine-doped tin oxide) films and must be wide for a convenient application and for a decreased ohmic resistance.

Another example of wide antennas is those incorporated in clothes. A wide choice of conductive materials exist but their resistance, e.g., in ITO films, is higher than that of copper.

Thanks to an expansion of the radiating surface, a low resistance can be achieved. However, the size is limited because the current paths from the supply to the upper limit should be approximately equal. The optimal width was determined via simulations (see Fig. 38). 


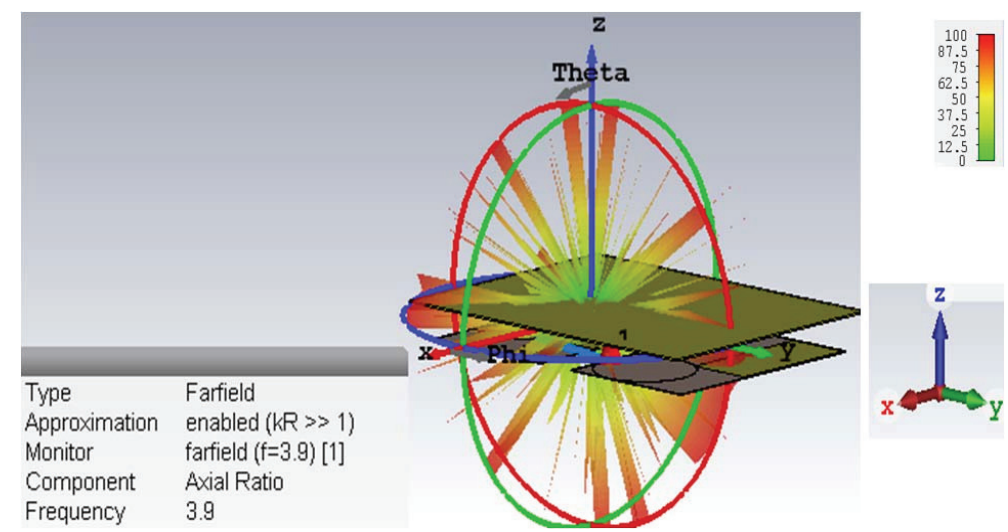

Fig. 36 Axial-ratio simulation results for antenna in Fig. 32.
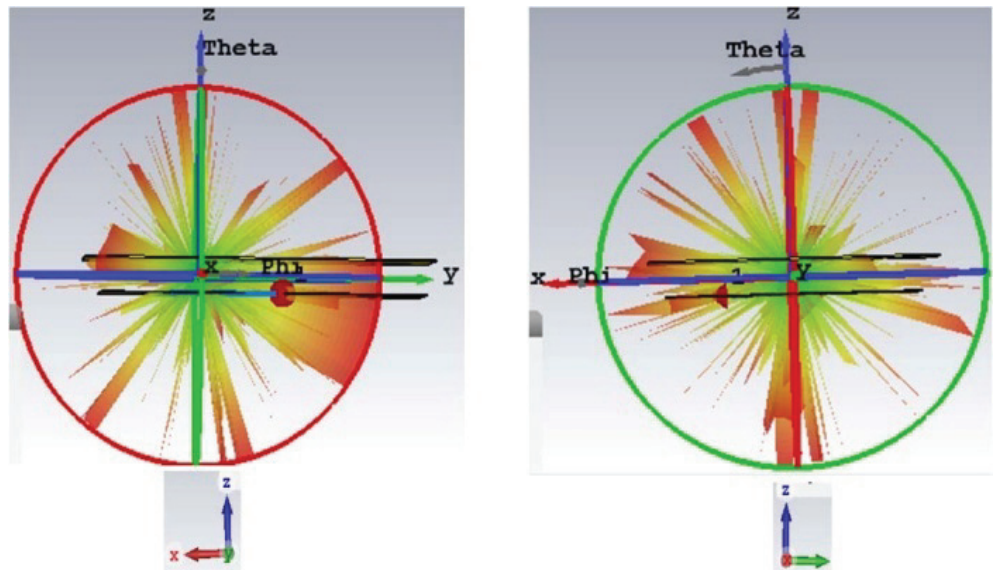

Fig. 37 Components on $\mathrm{X}$ and $\mathrm{Y}$ axes for antenna in Fig. 32, respectively.
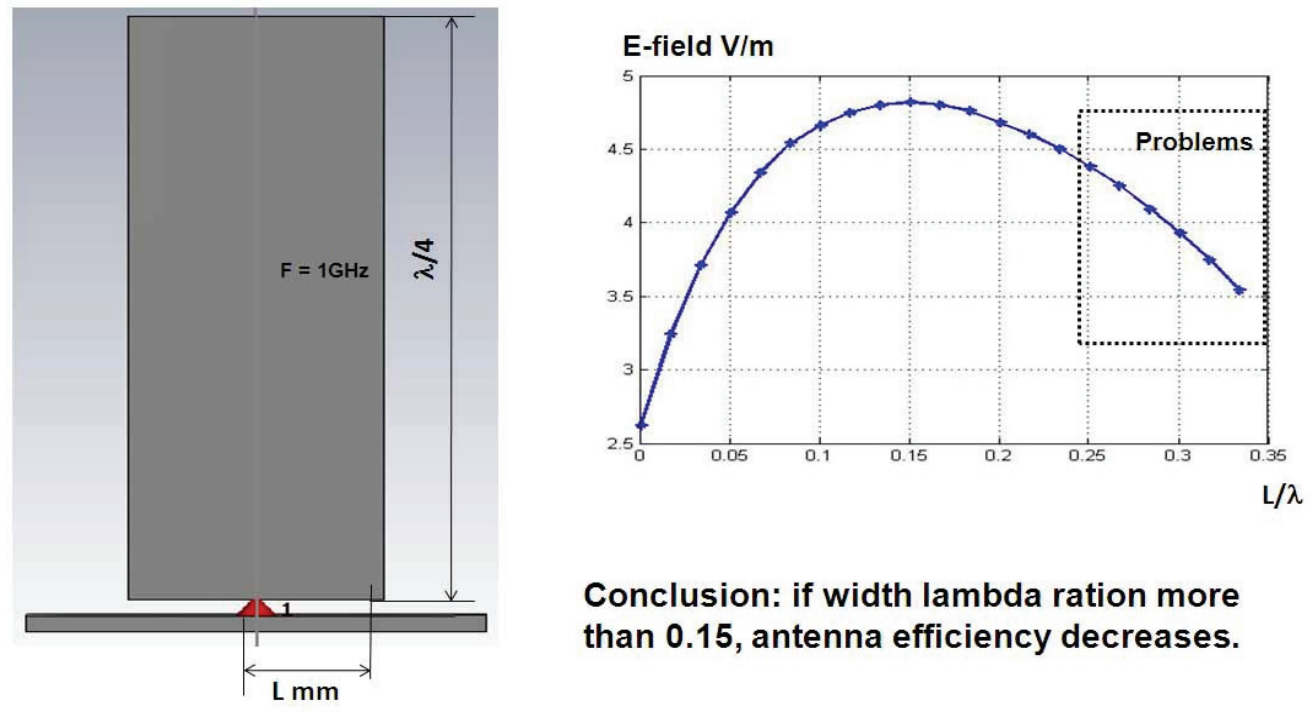

\section{Conclusion: if width lambda ration more than 0.15 , antenna efficiency decreases.}

Fig. 38 Dependence of antenna efficiency on radiator width.

One can see in Fig. 38 that the MB antenna model has a height of $L / 4$. Its wading $(L \mathrm{~mm})$ changes with ratio $L / \lambda$.
Regarding the right part in Fig. 38, after reaching its maximum at $L / \lambda=0.15$, the field radiation decreases. This decrease can be avoided if several lines of equal 


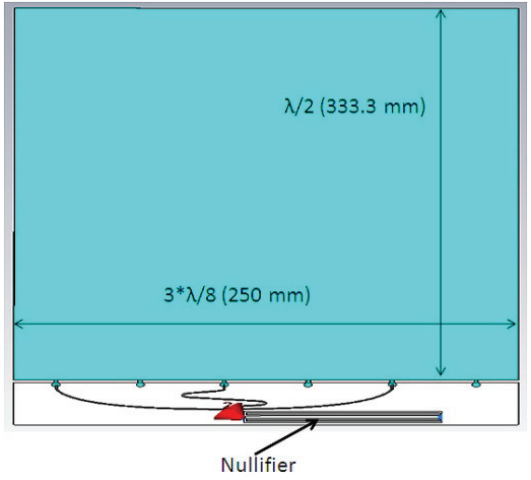

Fig. 39 Signal delivery with wide radiator. length are used to connect the antenna with the source and receiver. Fig. 39 presents such a radiator.

The respective simulation results are presented in Fig. 40.

In this study, a transparent antenna (Fig. 41) was produced and investigated in a laboratory chamber.

Moreover, measurements were conducted to compare the copper antenna and transparent antenna. According to Fig. 42, the desired results were obtained.

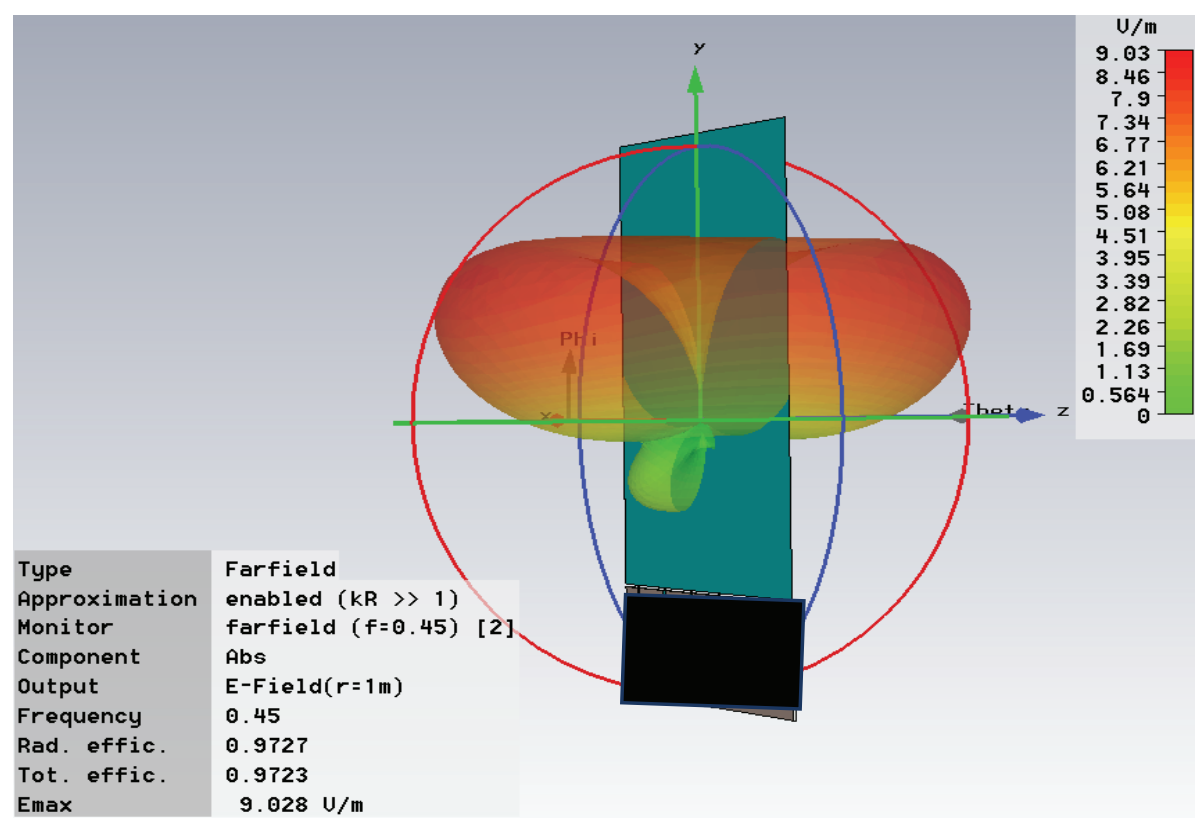

Fig. 40 Simulation results of transparent antenna.

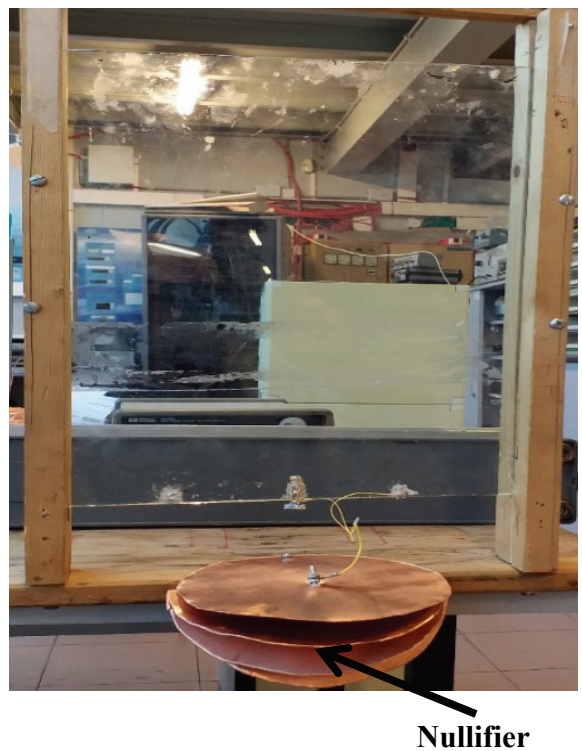

Fig. 41 Built and investigated transparent antenna in laboratory. 


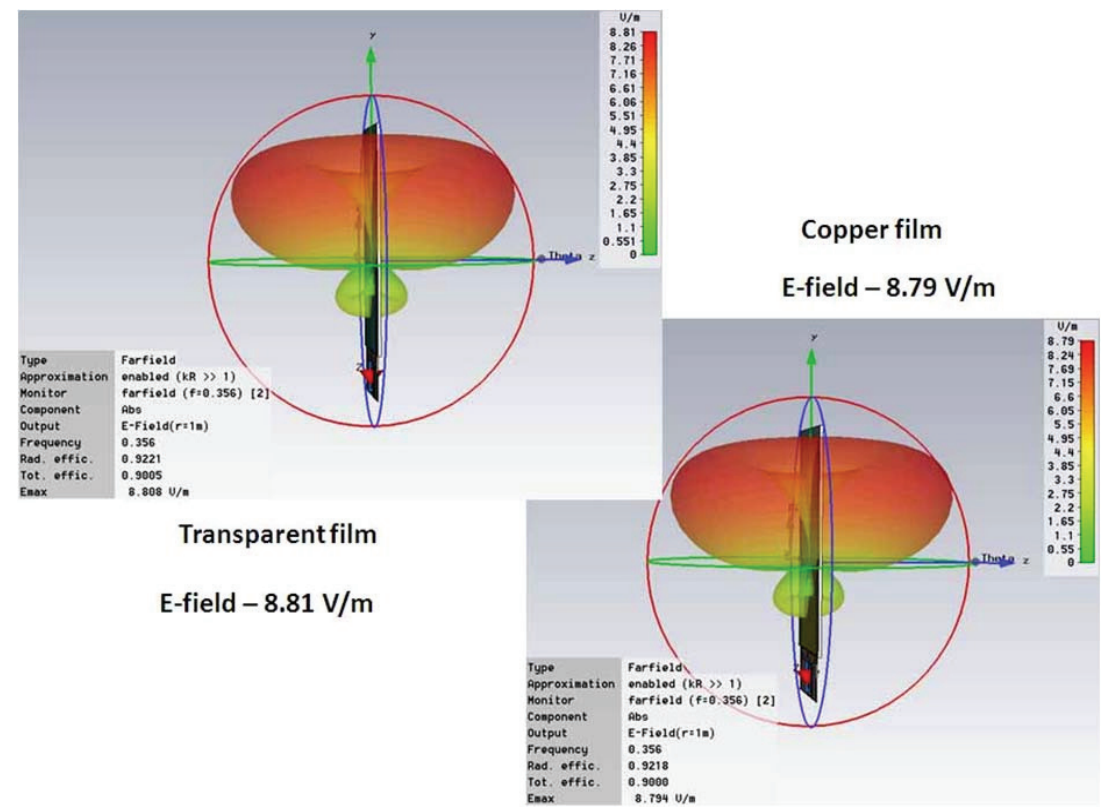

Fig. 42 Comparison of transparent and copper antennas.
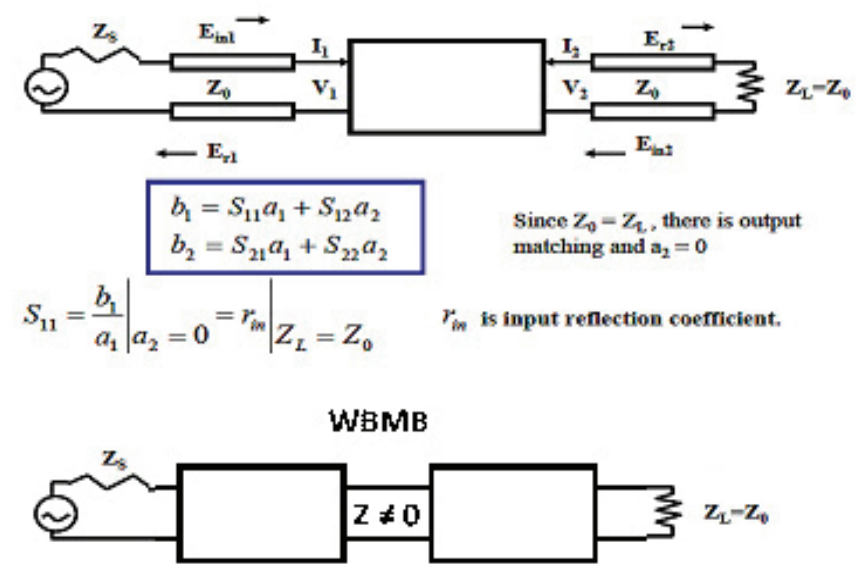

Fig. 43 S11 parameter measurement for single and compliment antennas.

\section{Very Wide Band Antennas}

Two important applications for broadband antennas exist: Firstly, systems with impulse modulation in which information is transmitted via short impulses (UWB systems). These systems need high frequencies and very wide band antennas. Secondly, antenna systems in modern and future cell phones must receive many frequencies. The presented WBMB antenna is a solution to these problems.

As usual, simulations were conducted. The main parameters of the WBMB antenna were the field density and total efficiency. Using the S11 parameters is inconvenient for complicated antennas.
The investigation resembles that of resonance systems. For single resonance circuits, the most used parameter is the contour quality factor (Q). However, for systems with several resonance circuits, parameters that characterize the complete system are employed, e.g., pass band, losses, and group delay.

If the load equals zero, the S11 parameters describe a four-terminal network. However, in a WBMB antenna, two four-terminal networks exist (see Figs. 43 and 44).

The frequency band of an antenna in a modern cell phone is $0.7-2.5 \mathrm{GHz}$. The proposed WBMB antenna achieves the necessary parameters at each frequency 
in this wide frequency band. A printed WBMB antenna is presented in Fig. 43.

A simulation of the field density at a distance of 1 $\mathrm{m}$ is presented in Fig. 45. The probe was located right from the antenna, at a distance of $1 \mathrm{~m}$.

The S21 parameters of this antenna were measured in an anechoic chamber. The arrangement is shown in Fig. 46.

Fig. 47 presents the measurement results.
According to the results, the antenna works at all frequencies of the cell phone system. The S21 parameters of several monopoles (see Fig. 48), tuned at different frequencies, were determined for this measurement.

The results of three tuned antenna monopole lengths are given in Fig. 49.

Consequently, to receive signals with a WBMB antenna, three monopoles must be employed. This is possible in cell phones.

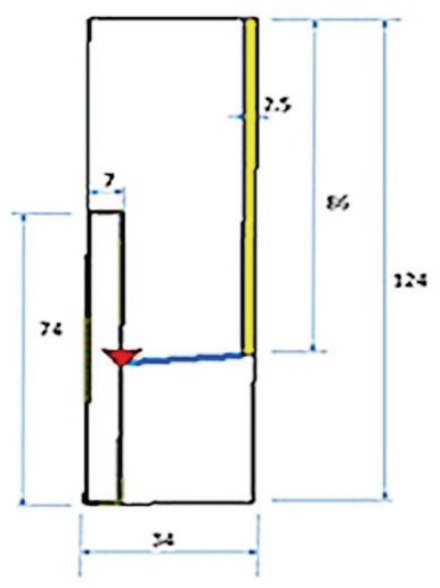

(a)

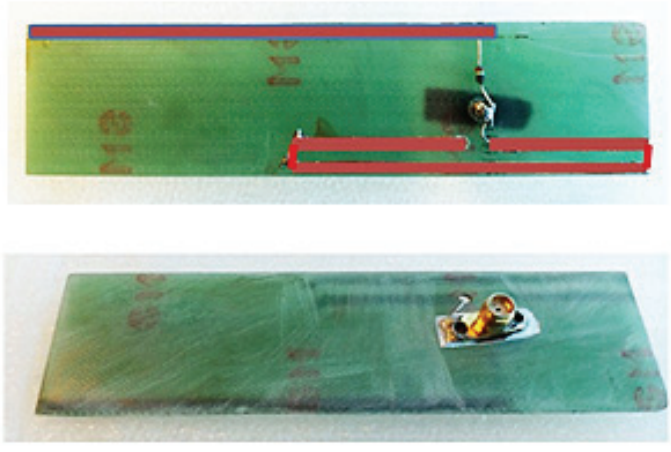

(b)

Fig. 44 (a) Construction and (b) photo of WBMB antenna for cell phone.

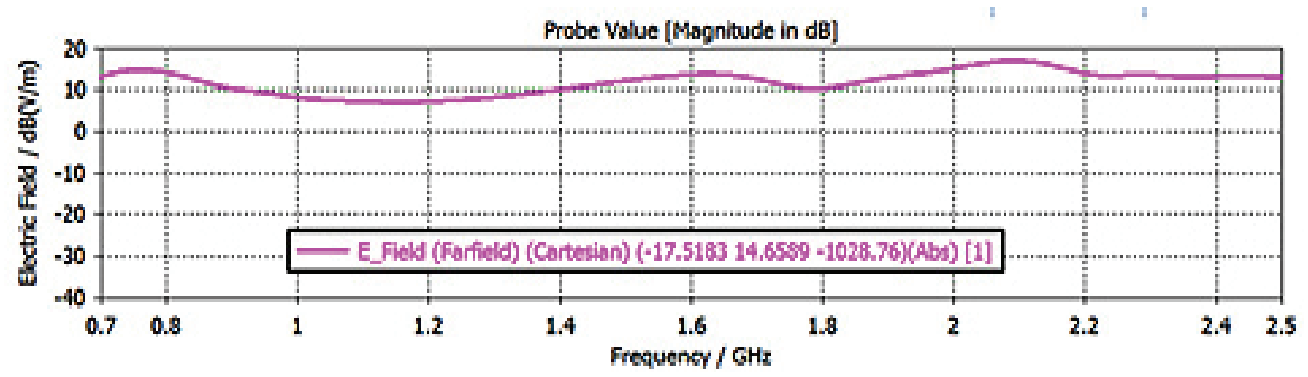

Fig. 45 Simulation of field density of antenna in Fig. 43.

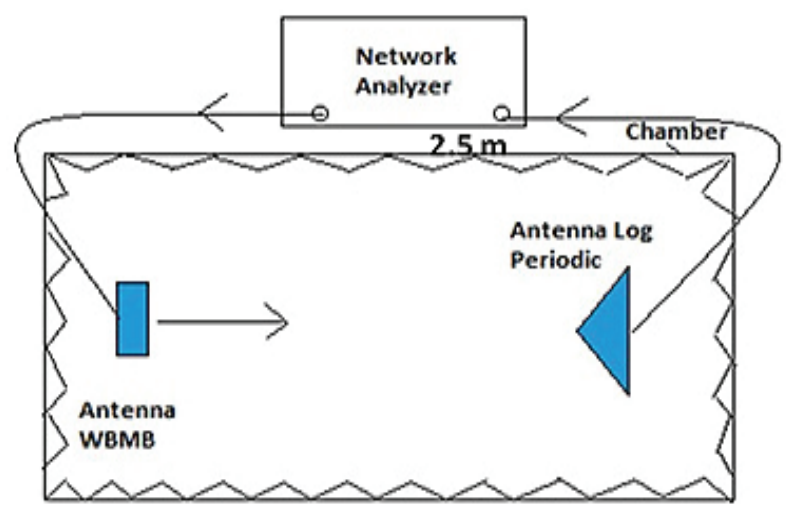

Fig. 46 Measurement of S21 parameters. 


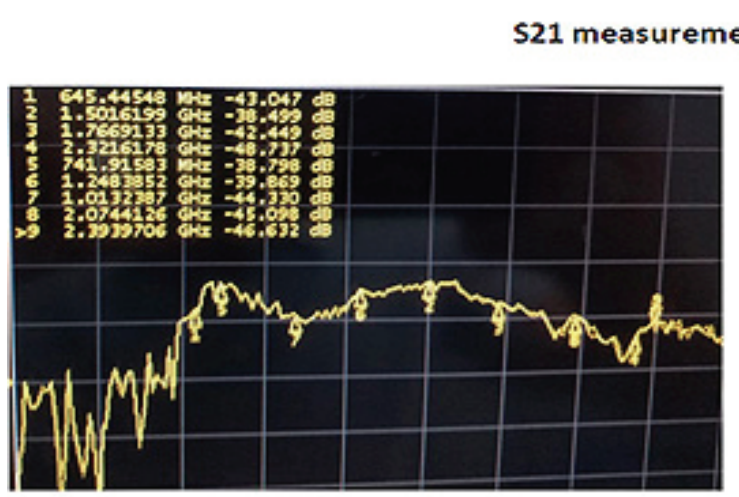

\begin{tabular}{|r|r|}
\hline Freg. $\mathrm{MHz}$ & S21, dB \\
\hline 645 & -43 \\
\hline 741 & -38 \\
\hline 1000 & -44 \\
\hline 1248 & -39 \\
\hline 1500 & -38 \\
\hline 1766 & -42 \\
\hline 2070 & -45 \\
\hline 2393 & -46 \\
\hline
\end{tabular}

Fig. 47 Results obtained with network analyzer.

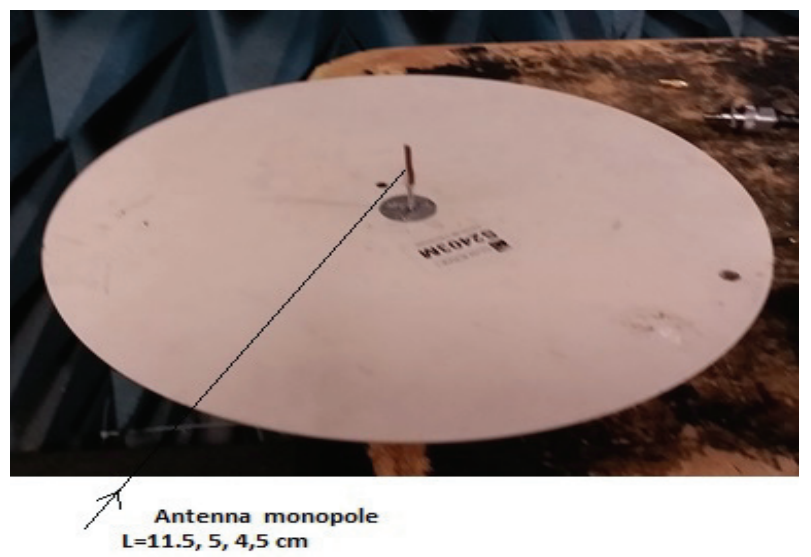

Fig. 48 Several antenna monopole lengths were investigated.

\begin{tabular}{|l|l|l|l|}
\hline Freq, $\mathrm{MHz}$ & $\begin{array}{c}\text { S21, dB } \\
\text { Monopole, } \\
\mathrm{L}=11.5 \mathrm{~cm}\end{array}$ & $\begin{array}{c}\text { S21, dB } \\
\text { Monopole, } \\
\mathrm{L}=5 \mathrm{~cm}\end{array}$ & $\begin{array}{c}\text { S21, dB } \\
\text { Monopole, } \\
\mathrm{L}=4.5 \mathrm{~cm}\end{array}$ \\
\hline 603 & -29 & -48 & -45 \\
\hline 729 & -34 & -45 & -45 \\
\hline 1000 & -39 & -35 & -42 \\
\hline 1272 & -38 & -37 & -39 \\
\hline 1495 & -37 & -37 & -35 \\
\hline 1760 & -47 & -38 & -35 \\
\hline 2026 & -49 & -41 & -37 \\
\hline 2225 & & -42 & -41 \\
\hline
\end{tabular}

Fig. 49 Comparison of S21 parameters of three monopole antenna lengths.

\section{Conclusion}

A new antenna type (patent number 9,419,327; patent number 9,685,704; patent number 10,050,353) with the following properties is proposed:

- It can be fed by a single line without grounding.

- It can be a wide-frequency band antenna.
- In case of a regular monopole instead of a ground, a nullifier can be used.

- It can be an antenna with a wide radiator.

- The antenna can be bent to decrease its height.

- The antenna can receive signals with circular polarization.

- It can be a wideband feed for a satellite antenna. 
- It can work with several signal sources simultaneously.

- It can be a solution for cell phones that need one antenna for all frequency bands.

Regarding a usual monopole antenna:

- The monopole height can be any if the reactance can be compensated and the active resistance of the antenna matches to source resistance.

- It is not necessary to use grounding. The second end of the source can be connected to the nullifier.

- The PCB cannot always be a GP. It depends on the perimeter of the $\mathrm{PCB}$.

\section{Acknowledgement}

The author thanks Professor M. Haridim and Dr. Tsingauz for taking part in the study and preparing the manuscript, engineer Rechels and programmer Shalyt for assisting in the preparations, and numerous students for the implementation of the simulations during the diploma project preparation.

\section{References}

[1] Bank, M. 2017. It Is Quite Another Electricity, Transmitting by One Wire and without Grounding (2nd ed.). Singapore: Partridge Publishing.

[2] Patent US 9419327 B2.

[3] Bank, M. 2016. Electrical energy transmission system with a single transmission line. US Patent 9,246,405 B2, Jan. 26, 2016.

[4] Bank, M. 2012. "New One-Way Line for Electric Transmission System." Journal of Energy and Power Engineering 6 (8): 1320-7.

[5] Haridim, M., and Bank, M. 2016. System for radiating radio frequency signals. US 9,419,327 B2 Aug. 16, 2016.

[6] Bank, M. 2003. "On Some Misunderstandings in Dipole and Monopole Using." IEEE Antenna and Propagation Magazine 45 (1): 143-7.

[7] Bank, M., Haridim, M., Tsingouz, V., and Ibragimov, Z. 2012. "Highly Effective Handset Antenna." Int'l. J. of Communications 6 (2): 80-7.

[8] Kalichman, S., and Levin, Y. 1965. Fundamentals of Theory and Calculation of the Broadcast Receivers Based on Semiconductors Instruments. Moscow: Communication. 